\title{
Hyperexpandable, self-healing macromolecular crystals with integrated polymer networks
}

\author{
Ling Zhang ${ }^{1}$, Jake B. Bailey ${ }^{1}$, Rohit H. Subramanian ${ }^{1}$, Alexander Groisman ${ }^{2}$, and F. Akif \\ Tezcan ${ }^{1,3,{ }^{*}}$ \\ ${ }^{1}$ Department of Chemistry and Biochemistry, University of California, San Diego, La Jolla, CA, \\ USA. \\ 2Department of Physics, University of California, San Diego, La Jolla, CA, USA. \\ ${ }^{3}$ Materials Science and Engineering, University of California, San Diego, La Jolla, CA, USA.
}

\section{Abstract}

The formation of condensed matter typically involves a tradeoff between structural order and flexibility. As the extent and directionality of interactions between atomic or molecular components increase, materials generally become more ordered but less compliant, and vice versa. Nevertheless, high levels of structural order and flexibility are not necessarily mutually exclusive; there are many biological (such as microtubules ${ }^{1,2}$, flagella ${ }^{3}$, viruses ${ }^{4,5}$ ) and synthetic assemblies (for example, dynamic molecular crystals ${ }^{6-9}$ and frameworks ${ }^{10-13}$ ) that can undergo considerable structural transformations without losing their crystalline order and that have remarkable mechanical properties ${ }^{8,14,15}$ that are useful in diverse applications, such as selective sorption ${ }^{16}$, separation ${ }^{17}$, sensing ${ }^{18}$ and mechanoactuation ${ }^{19}$. However, the extent of structural changes and the elasticity of such flexible crystals are constrained by the necessity to maintain a continuous network of bonding interactions between the constituents of the lattice. Consequently, even the most dynamic porous materials tend to be brittle and isolated as microcrystalline powders ${ }^{14}$, whereas flexible organic or inorganic molecular crystals cannot expand without fracturing. Owing to their rigidity, crystalline materials rarely display self-healing behaviour ${ }^{20}$. Here we report that macromolecular ferritin crystals with integrated hydrogel polymers can isotropically expand to 180 per cent of their original dimensions and more than 500 per cent of their original volume while retaining periodic order and faceted Wulff morphologies. Even after the separation of

Reprints and permissions information is available at http://www.nature.com/reprints.

* tezcan@ucsd.edu. Correspondence and requests for materials should be addressed to F.A.T. Author contributions

L.Z., J.B.B. and F.A.T conceived the work and designed the experiments. L.Z. conducted the majority of the experiments and performed light microscopy analysis. L.Z. and J.B.B. performed XRD and SAXS analyses. L.Z. and R.H.S. performed confocal microscopy analysis. A.G. designed the microfluidic cells. F.A.T wrote the manuscript with contributions from all authors.

Online content

Any Methods, including any statements of data availability and Nature Research reporting summaries, along with any additional references and Source Data files, are available in the online version of the paper at https://doi.org/10.1038/s41586-018-0057-7.

Competing interests

L.Z., J.B.B. and F.A.T. have submitted a patent application based on the work described here.

Extended data is available for this paper at https://doi.org/10.1038/s41586-018-0057-7.

Supplementary information is available for this paper at https://doi.org/10.1038/s41586-018-0057-7.

Publisher's note: Springer Nature remains neutral with regard to jurisdictional claims in published maps and institutional affiliations. 
neighbouring ferritin molecules by 50 ångströms upon lattice expansion, specific molecular contacts between them can be reformed upon lattice contraction, resulting in the recovery of atomic-level periodicity and the highest-resolution ferritin structure reported so far. Dynamic bonding interactions between the hydrogel network and the ferritin molecules endow the crystals with the ability to resist fragmentation and self-heal efficiently, whereas the chemical tailorability of the ferritin molecules enables the creation of chemically and mechanically differentiated domains within single crystals.

Hydrogel polymers present a stark contrast to molecular crystals in that they lack structural order, but are highly elastic and adaptive, can expand considerably and self-heal when equipped with dynamic bonding functionalities ${ }^{21,22}$. Previously, the isotropic swelling -contraction behaviour of hydrogels has been used to modulate the lattice spacing of colloidal nanoparticle arrays ${ }^{23}$, and recently, to expand biological tissue samples and thus facilitate high-resolution fluorescence imaging ${ }^{24}$. In this study, we examine whether the mechanical properties of hydrogels could be endowed upon molecular crystals. That is, can crystal lattices that are formed by discrete molecules that are connected via specific bonding interactions be mechanically modulated through the integration of polymeric hydrogels? To create hydrogel-expandable molecular crystals, we surmised that the following design parameter conditions should be met: (1) lattices should be mesoporous to enable the hydrogel network to penetrate efficiently and uniformly into the crystals; (2) intermolecular interactions between the constituents of the lattices should be reversible and chemically specific (that is, contain directional and dynamic bonds), such that they disengage with ease during expansion and re-engage with high fidelity upon contraction; (3) interactions between the constituents of the lattice and the hydrogel network should be extensive to maintain the integrity of the crystal-polymer hybrid at all times and sufficiently dynamic to minimize the build-up of local strain and to enable self-healing.

With these parameters in mind, we arrived at hybrid materials composed of ferritin crystals integrated with the superabsorbent poly(acrylate-acrylamide), or p(Ac-Am), copolymer hydrogels, whose swelling-contraction behaviour can be modulated by the ionic strength and $\mathrm{pH}^{25}$. Ferritin is a 24-meric, quasi-spherical protein with 432 symmetry, an outer diameter of $12 \mathrm{~nm}$, an inner diameter of $8 \mathrm{~nm}$, and a molecular weight ${ }^{26}$ of more than 500,000 Da. Human heavy-chain ferritin forms highly ordered, face-centred cubic (fcc) crystals that routinely grow to more than $200 \mu \mathrm{m}$ in size and diffract to less than $2.0 \AA$ A . The fcc lattice (Fig. 1a) is characterized by a mesoporous network consisting of cube-shaped, 6-nm-wide chambers (Fig. 1b) that are interconnected by smaller, octahedron-shaped cavities that taper to a pore size of about $2 \mathrm{~nm}$ at their narrowest (Fig. 1c), thus fulfilling condition (1). The lattice is formed through highly specific, metal-mediated contacts between neighbouring ferritin molecules (Fig. 1d), which are promoted through the K86Q surface mutation to enable metal coordination ${ }^{27}$. The absence of any other interprotein contacts means that the entire lattice bonding framework of ferritin molecules can be formed or broken via binding or removal of metal ions (such as $\mathrm{Ca}^{2}+$ ), satisfying condition (2). Finally, ferritin bears a small negative charge, with a zeta potential ranging from $-5.5 \mathrm{mV}$ at $\mathrm{pH} 6.0$ to $-7.3 \mathrm{mV}$ at pH 7.5 (Extended Data Fig. 1a, b). The exterior surface of ferritin presents a diffuse distribution of both negatively and positively charged residues (Extended Data Fig. 1c), 
which should enable uniform association with the $\mathrm{p}(\mathrm{Ac}-\mathrm{Am})$ network through a combination of ionic and H-bonding interactions, thus fulfilling condition (3) (Fig. 1e).

We first examined the efficiency of molecular diffusion and polymerization within ferritin crystals. Diffusion into single ferritin crystals was assessed using the fluorescent tracer rhodamine B by confocal fluorescence microscopy experiments. These experiments showed that a typical crystal (edge length, $\boldsymbol{l}_{\text {edge }}=50-250 \mu \mathrm{m}$ ) was completely infiltrated by rhodamine B (Extended Data Fig. 2a), which is considerably larger $\left(479 \mathrm{~g} \mathrm{~mol}^{-1}\right)$ than the Ac and Am molecules (both $71 \mathrm{~g} \mathrm{~mol}^{-1}$ ), within $15 \mathrm{~min}$. In a typical preparation of crystalhydrogel hybrids, ferritin crystals were incubated with polymer precursors $(8.625 \%(\mathrm{w} / \mathrm{v})$ sodium acrylate, $2.5 \%$ acrylamide and $0.2 \% \mathrm{~N}, \mathrm{~N}$ '-methylenebis(acrylamide)) for at least 10 $\mathrm{h}$ to ensure their uniform distribution in the lattice interstices. This treatment caused no apparent damage to the crystals (see Supplementary Information for quantification of polymer precursor concentrations inside the crystals). Crystals were then transferred into a solution containing $1 \%(\mathrm{w} / \mathrm{v})$ ammonium persulfate (APS) and $1 \%(\mathrm{v} / \mathrm{v})$ tetramethylethylenediamine (TEMED) to initiate free-radical polymerization within the lattice, as well as $4 \mathrm{M}$ sodium chloride $(\mathrm{NaCl})$ to limit swelling during polymerization (Fig. 1e). To assess the kinetics of polymerization inside the crystals, we added $0.3 \%(w / v) 8$ hydroxypyrene-1,3,6-trisulfonic acid (pyranine) to the aforementioned co-monomer mixture. Pyranine has been reported to become covalently incorporated into the polymer backbone upon radical-mediated crosslinking and undergo a shift ${ }^{28}$ in its emission maximum from 512 to $420 \mathrm{~nm}$. Thus, the extent of in crystallo polymerization could be monitored through the decrease of green fluorescence intensity (emission wavelength $\lambda_{\text {emission }}=500-550 \mathrm{~nm}$, excitation wavelength $\lambda_{\text {excitation }}=488 \mathrm{~nm}$ ), indicating that hydrogel formation was complete in less than 2 min for a crystal with $\boldsymbol{l}_{\text {edge }}=70 \mu \mathrm{m}$ (Extended Data Fig. 2b, c and Supplementary Video 1; see Extended Data Fig. 3 for polymer quantification via ${ }^{19}$ F nuclear magnetic resonance, NMR). Polymerization was promptly followed by intrusion of the aqueous $\mathrm{NaCl}$ solution into the crystal-hydrogel matrix, which was clearly visualized owing to the difference between the refractive indices of the salt solution $\left(\boldsymbol{n}_{\mathrm{D}}=1.3676\right)$ and the matrix ( $\mathrm{n}_{\mathrm{D}} \approx 1.34$ ) (Extended Data Fig. $2 \mathrm{~b}$ and Supplementary Videos 1,2 ). The solvent permeation process typically finished within $10 \mathrm{~min}$ and was accompanied by a small but noticeable enlargement of the crystals ( $\mathbf{5 \%}$ increase in edge length) (Extended Data Fig. 2c).

Full expansion of hydrogel-infused ferritin crystals was initiated by placing them in deionized water. As observed using light microscopy, the expansion of the crystals was highly isotropic and their sharply faceted, polyhedral morphologies were preserved even after they grew to $\geq 210 \%$ of their original dimensions (Fig. 2a and Supplementary Video 2; see Extended Data Fig. 4a and $\mathrm{b}$ for additional examples), often without the appearance of any defects. The expansion kinetics was biphasic, with time constants $\boldsymbol{\tau}_{\text {fast }}<100 \mathrm{~s}$ and $\boldsymbol{\tau}_{\mathrm{s}} \imath_{\mathrm{ow}} » 300 \mathrm{~s}$ (Fig. 2a). Isotropic growth continued indefinitely, until the edges of the materials were not discernible, but we typically stopped the process after $<10 \mathrm{~min}$, when considerable expansion had already occurred. No substantial release of ferritin molecules from the lattices was evident during the first 50 min of expansion (Extended Data Fig. 4c). Addition of a concentrated monovalent salt solution $(\mathrm{NaCl}$ or $\mathrm{KCl})$ led to rapid dehydration 
and isotropic contraction of the expanded crystals to nearly their original size (Fig. 2a and Extended Data Fig. 5a). Recovery of the original crystal dimensions could be achieved by further addition of $\mathrm{CaCl}_{2}$, owing to the ability of $\mathrm{Ca}^{2+}$ to both screen the negatively charged polymer backbone more effectively and to re-engage specific interactions between ferritin molecules. The same effect was observed with other divalent metal-ion salts (Extended Data Fig. 5b). The expansion-contraction cycle could be repeated at least eight times without apparent loss in amplitude and change in crystal morphology when a monovalent metal-salt solution was used to induce contraction (Extended Data Fig. 6). We observed that crystals contracted with $\mathrm{CaCl}_{2}$ displayed considerably smaller expansion owing to the enhanced strength of the polymer network and protein-protein interactions. In control experiments, we examined other hydrogel formulations, including hydrogels that only contained polar but non-charged (pAm or poly-tris(hydroxymethyl)methyl(acrylamide)) or non-polar (poly-Nisopropylacrylamide) side-chains (Extended Data Fig. 7). All of these polymers led to either dissolution or disintegration of crystals after initiation of in crystallo polymerization, suggesting a lack of substantial interactions between the functional groups on these polymers and on the ferritin surface.

Interestingly, pAc hydrogels promoted isotropic expansion of the crystals in the absence of Am co-monomers (Extended Data Fig. 7), indicating that carboxylate side-chains are the primary mediators of interactions with ferritin molecules. By contrast, treatment of ferritin crystals with pre-formed pAc polymers, which cannot diffuse into the lattice, led to crystal dissolution upon transfer into water (Extended Data Fig. 7c). Together, these observations confirm that (i) there are extensive non-covalent interactions between ferritin molecules and the $\mathrm{p}(\mathrm{Ac}-\mathrm{Am})$ hydrogel matrix that preserve the structural integrity of even highly expanded crystals, and (ii) the hydrogel matrix continuously and uniformly pervades the entire lattice, thus promoting cooperative transmission of any lattice deformations to enable isotropic expansion-contraction.

We investigated the expansion-related changes in the lattice arrangement of ferritin molecules using small-angle X-ray scattering (SAXS). Initial experiments entailed bulk measurements of a large number of ferritin crystals suspended in a capillary tube. Figure $2 b$ shows the evolution of the 'powder' SAXS pattern of more than 100 single $\mathrm{p}(\mathrm{Ac}-\mathrm{Am})$ infused crystals upon the initiation of polymerization though the addition of APS/TEMED in a solution that contains no salt; thus, polymerization is immediately followed by expansion (see Methods for experimental details). The spectrum of the unexpanded crystals is indicative of an fcc lattice with a unit-cell parameter of $\boldsymbol{a}=182.40 \AA$. The isotropic growth of the unit cell is evident from the correlated shifts of the Bragg peaks to lower angles. The decay of the higher-angle peaks is considerably more rapid and is accompanied by the emergence of the ferritin form factor. This is consistent with the picture that as the crystal expands, the hydrogel matrix becomes less dense around the ferritin molecules, leading to their increased mobility. However, the (111) reflection is still evident after $20 \mathrm{~min}$ of expansion, which means that some long-range periodic order is still present when the unit cell has grown to $\boldsymbol{a}=325 \AA$ (Fig. 2c) and the volume of the material has increased to $570 \%$ of its original value. 
To probe the reversibility of lattice expansion, we set up a micro-fluidic flow cell for singlecrystal SAXS experiments (Extended Data Fig. 8), which circumvent the inherent issues associated with bulk measurements in a small capillary tube (such as sample heterogeneity and inefficient solvent diffusion). The SAXS data in Fig. $2 d$ indicate that a single-crystal lattice that has expanded by $27 \%$ - corresponding to a separation of $35 \AA$ between neighbouring ferritin molecules - can return to its original dimensions upon $\mathrm{NaCl} / \mathrm{CaCl}_{2^{-}}$ induced contraction. To examine whether this recovery also occurs at the level of atomic periodicity, we conducted high-angle, single-crystal X-ray diffraction (XRD) experiments at room temperature (Fig. 3a-c). These experiments showed that crystals that expanded by up to $40 \%$ could fully regain their native diffraction pattern upon contraction with divalent metal-ion salts (Extended Data Fig. 5b). With such expanded and Ca-contracted crystals, we consistently obtained datasets with resolutions $<1.15 \AA$ at a synchrotron source at $100 \mathrm{~K}$ (Extended Data Table 1). Interestingly, the resulting crystal structures revealed two different conformational states of the $\mathrm{Ca}^{2+}$-bridged ferritin-ferritin interfaces (Fig. 3d): about $60 \%$ of these interfaces were found in the native configuration (as shown in Fig. 1d, but with a well resolved Ca-coordinated water molecule), whereas the remaining $40 \%$ presented an alternative coordination mode for $\mathrm{Ca}^{2+}$, probably stemming from lattice rearrangements during contraction. Notably, the 1.06-Å-resolution crystal structure (R-factors, $\boldsymbol{R}_{\text {work }}=$ $9.10 \% ; \boldsymbol{R}_{\text {free }}=10.26 \%$; estimated coordinate error (dispersion precision indicator), $0.011 \AA$ ) is the highest-resolution ferritin structure reported until now. Our findings suggest that hydrogel infusion and the expansion-contraction process do not diminish XRD data quality and may actually improve it.

Any local anisotropy developed during the expansion or contraction of the hydrogel matrix would be expected to cause dislocations in the embedded ferritin lattice. Indeed, exposure of hydrogel-infused crystals to rapid changes or temporary spatial gradients in $\mathrm{NaCl}$ or $\mathrm{CaCl}_{2}$ concentrations frequently led to fracturing (see, for example, Fig. $3 \mathrm{~b}$ inset). However, these materials showed a remarkable ability to self-heal, whereby the cracks were spontaneously and, in some cases, scarlessly sealed (Fig. 4a, b and Supplementary Video 3), owing to the reversible bonding interactions of the hydrogel network with the protein molecules (Fig. 4c). It is important to note that covalently crosslinked hydrogels like $\mathrm{p}(\mathrm{Ac}-\mathrm{Am})$ do not typically self-heal unless they are modified with dynamic bonding functionalities ${ }^{22,29}$. In the case of our materials, the role of such functional groups is fulfilled by the ferritin molecules, which act as interaction hubs for polymer chains. During expansion-contraction cycles, cracks tended to reoccur in the same loci in a given crystal (Supplementary Video 3). This observation suggests that the healed interfaces had not fully regained the original hydrogel crosslinking density of the bulk material, at least in the time scale (several minutes) of the experiments. Hydrogel integration substantially mitigated the brittleness of native ferritin crystals (Extended Data Fig. 9a). We observed no fragmentation, even in cases of substantial fracturing that propagated throughout the crystals, and fissures as wide as $20 \mu \mathrm{m}$ could be closed to recover near-native crystal morphology (Fig. 4b). The ferritin crystal-hydrogel hybrids had a reduced modulus of about $1 \mathrm{GPa}$, which is similar to that of ferritin crystals (Extended Data Fig. 9b), but several orders of magnitude higher than those of hydrogels ${ }^{30}$. The hybrids are also highly thermostable, maintaining their crystalline order at $280^{\circ} \mathrm{C}$ (Extended Data Fig. 9c). 
Owing to the inherent chemical tailorability of ferritin molecules, the crystal-hydrogel hybrids could be functionalized in different ways. They could be constructed from ferritin molecules with mineralized ferrihydrite in their interior cavity (Extended Data Fig. 9d and Supplementary Video 4), thus exploiting ferritin's native function as a ferroxidase, or with fluorescent tags covalently attached to their exterior (Fig. 4d, e and Supplementary Video 4). Additionally, spatially differentiated, core-shell crystals were created using a layer-by-layer growth method (Fig. 4d, e). When infused with p(Ac-Am), such nanoparticle- or fluorophore-functionalized lattices displayed the same isotropic expansion-contraction behaviour as non-functionalized ones. The layer-by-layer growth process was further modified whereby the core lattice domain (labelled with rhodamine groups) was first covalently fixed through the chemical crosslinking of ferritin molecules with glutaraldehyde, followed by the growth of an uncrosslinked, unlabelled shell layer and the incorporation of the $\mathrm{p}(\mathrm{Ac}-\mathrm{Am})$ polymer into the composite lattice. Hydration of such 'fixed core/expandable shell' crystals led to complete fragmentation of the shell layer due to the strain generated at the mechanically mismatched core-shell interface, exposing the morphologically unaltered core layer (Fig. 4e). These examples highlight the facility with which chemical and mechanical patterning are achieved in protein crystal-hydrogel hybrids.

We have reported here a new form of materials that integrate macro-molecular protein crystals with synthetic polymer networks. These hybrids seamlessly combine the structural order and periodicity of crystals, the adaptiveness and tunable mechanical properties of polymeric networks and the chemical versatility of protein building blocks. Additionally, the ability to reversibly expand-contract crystal lattices and mobilize their protein components may provide a new means to improve XRD quality and explore otherwise inaccessible protein structural states using three-dimensional protein crystallography. Protein crystals are often highly porous, sometimes containing up to $90 \%$ solvent, and are usually assembled through weak, non-covalent packing interactions; therefore, our approach should be applicable to other protein lattices. Their potential for generalizability, coupled with the chemical tailorability of synthetic polymers and the genetic mutability of proteins, should make protein crystal-hydrogel hybrids a rich medium for materials science.

\section{METHODS}

\section{Protein expression, purification and characterization.}

The plasmid for the $\Delta \mathrm{C}^{*}$ variant of human heavy-chain ferritin (HuHF), devoid of all native cysteine residues (C90E, C102A and C130A), was obtained via site-directed mutagenesis as previously described ${ }^{31}$. Expression and purification of $\Delta \mathrm{C}^{*}$ was performed according to the previously published protocol ${ }^{32}$.

\section{Determination of zeta potentials.}

Purified ferritin was concentrated to about $200 \mu \mathrm{M}$ and exchanged into a buffer solution containing $50 \mathrm{mM}$ 2,2-Bis(hydroxymethyl)-2,2/,2//-nitrilotriethanol (Bis-Tris) (pH 6.0), 50 mM 4-(2-hydroxyethyl)-1-piperazineethanesulfonic acid (HEPES) (pH 7.0) or $50 \mathrm{mM}$ HEPES ( $\mathrm{pH}$ 7.5) using an Amicon Ultra centrifugal filter unit (10 kDa cutoff). The zeta potentials of ferritin in the three different buffers were determined using a Zetasizer Nano 
ZS90 (Malvern Instruments). Experimental runs were performed to collect 12 datasets with a He-Ne laser at $633 \mathrm{~nm}$.

\section{Formation of crystal-hydrogel hybrids.}

Polymer precursor solution. $25 \mathrm{mM}$ (HEPES; $\mathrm{pH} 7.0), 30 \mathrm{mM} \mathrm{CaCl}_{2}, 917 \mathrm{mM}(8.625 \%$ w/v) sodium acrylate, $352 \mathrm{mM}(2.5 \% \mathrm{w} / \mathrm{v})$ acrylamide and $13 \mathrm{mM}(0.2 \% \mathrm{w} / \mathrm{v}) \mathrm{N}, \mathrm{N}^{;}-$ methylenebis(acrylamide). Polymerization solution. $4 \mathrm{M} \mathrm{NaCl}, 1 \%(\mathrm{w} / \mathrm{v})$ APS and $1 \%(\mathrm{v} / \mathrm{v})$ TEMED. Octahedron-shaped ferritin crystals formed over 1-2 days in a buffered solution containing $25 \mathrm{mM}$ HEPES (pH 7.0), 3-14.5 $\mu \mathrm{M}$ protein (per 24meric ferritin cage) and 4.5$7.5 \mathrm{mM} \mathrm{CaCl}_{2}$. Once the ferritin crystals matured, the crystallization solution was replaced with the polymer precursor solution. Crystals were soaked for more than $10 \mathrm{~h}$ to ensure full infusion of the monomers into the ferritin crystals, and were then individually transferred with a mounted CryoLoop (Hampton) to the polymerization solution for 5 min, initiating in crystallo polymerization. Alternatively, the crystallization solution was replaced with the polymerization solution for the bulk polymerization of many crystals at once.

\section{Measurement of the rate of diffusion into ferritin crystals.}

A large ferritin crystal was transferred with a mounted CryoLoop onto a glass slide, and 20 $\mu \mathrm{l}$ of a solution containing $20 \mu \mathrm{M}\left(0.01 \mathrm{mg} \mathrm{ml}^{-1}\right)$ rhodamine $\mathrm{B}, 30 \mathrm{mM} \mathrm{CaCl}_{2}$ and $25 \mathrm{mM}$ HEPES ( $\mathrm{pH}$ 7.0) was added to the crystal. The rhodamine diffusion process was monitored with a $10 \times$ air objective installed on a spinning-disk confocal Axio Observer inverted microscope (Zeiss) equipped with a pair of Quantum 5125C cameras (Roper), using a filter to collect light at 575-650 nm (red channel). Differential interference contrast (DIC) and fluorescence (564 nm excitation) images were captured at 1-s intervals with a 10-ms exposure. Images were collected in Slidebook 6 (Intelligent Imaging Innovations) and analysed using Fiji (http://fiji.sc/Fiji).

\section{Determination of in crystallo polymerization dynamics.}

Ferritin crystals were incubated in a polymer precursor solution supplemented with $5.7 \mathrm{mM}$ $(0.3 \%)$ pyranine (Sigma-Aldrich). After $12 \mathrm{~h}$, an individual crystal was transferred onto a glass slide and polymerization was initiated by adding $10 \mu$ of the polymerization solution. Hydrogel polymerization throughout the crystal and the corresponding decrease of pyranine fluorescence were monitored with a $20 \times$ air objective on the confocal microscope as described above, using a filter to collect light at 500-550 $\mathrm{nm}$ (green channel). DIC and fluorescence (488 nm excitation) images were captured at 1-s intervals with 100-ms (DIC) and 1-s (fluorescence) exposures.

\section{Scanning electron microscopy of ferritin crystals.}

Native ferritin crystal and crystal-hydrogel hybrid samples were deposited onto glowdischarged, Formvar/carbon-coated Cu grids (Ted Pella Inc.). Each grid was blotted with filter paper to remove excess liquid. Grids were mounted onto a STEM 12x v2 sample holder and imaged using a Sigma 500 scanning electron microscope (Zeiss) at an accelerating voltage of $1 \mathrm{kV}$ using a $30-\mu \mathrm{m}$ aperture. 


\section{Polymer quantification with ${ }^{19} \mathrm{~F}$ NMR.}

Large-scale crystallization of ferritin was carried out in a 24-well culture plate (Costar). 100 $\mu \mathrm{l}$ of $25 \mu \mathrm{M}$ ferritin in $15 \mathrm{mM}$ Tris (pH 7.4) and $150 \mathrm{mM} \mathrm{NaCl}$ ) was combined with $100 \mu \mathrm{l}$ of a buffered solution containing $50 \mathrm{mM}$ HEPES (pH 7.0) and $12 \mathrm{mM} \mathrm{CaCl}_{2}$. Crystals formed overnight and matured over $72 \mathrm{~h}$. The solution in each well was replaced with $100 \mu \mathrm{l}$ of a polymer precursor soaking solution containing: $25 \mathrm{mM}$ HEPES (pH 7.0), $30 \mathrm{mM}$ $\mathrm{CaCl}_{2}, 179.9 \mathrm{mM}$ 2-(trifluoromethyl)acrylic acid, $744.8 \mathrm{mM}$ sodium acrylate, $350.7 \mathrm{mM}$ acrylamide and $20.4 \mathrm{mM} \mathrm{N}, \mathrm{N}$ '-methylenebis(acrylamide). After soaking overnight, this solution was removed, and the crystals were washed with a buffered solution $(25 \mathrm{mM}$ HEPES, $\mathrm{pH} 7.0 ; 30 \mathrm{mM} \mathrm{CaCl}_{2}$ ) to remove unincorporated monomers. Polymerization was initiated by replacing the washing solution with $100 \mu \mathrm{l}$ of the polymerization solution. After $10 \mathrm{~min}$, the crystals were transferred into an Eppendorf tube and centrifuged at 2,000g for 60 $\mathrm{s}$. The supernatant was decanted, and the crystals were resuspended in $1 \mathrm{ml} \mathrm{D}_{2} \mathrm{O}$. Concentrated $\mathrm{HCl}$ was added until the $\mathrm{pH}$ of the solution was approximately 4.0 to facilitate crystal decomposition. $705 \mu \mathrm{l}$ of this solution was transferred into an NMR tube and supplemented with $4.6 \mathrm{mM}$ trifluoroacetic acid. The ${ }^{19} \mathrm{~F}-\mathrm{NMR}$ spectrum was collected using a 300M Bruker AVA spectrometer with a ${ }^{19} \mathrm{~F}$ probe (Extended Data Fig. 3). The peak at -64.94 p.p.m. corresponds to free 2-(trifluoromethyl)acrylic acid, the cluster of peaks near -67.07 p.p.m. to 2-(trifluoromethyl)acrylic acid that has been incorporated into the polymer, and the peak at -75.51 p.p.m. to the trifluoroacetic acid standard. From the integration of these peaks it was deduced that (a) the total concentration of 2-(trifluoromethyl)acrylic acid in the solution was $8.2 \mathrm{mM}$ and (b) $74.7 \%$ of the monomeric precursor was incorporated into the polymer matrix inside the crystals. The protein concentration was determined to be 60.0 $\mu \mathrm{M}$ using the Bradford assay ${ }^{33}$, and the molar ratio of 2-(trifluoromethyl) acrylic acid to ferritin was calculated as 137:1. Given this ratio and the fact that each unit cell of the ferritin crystals contains four ferritin cages and has a volume of about $5832 \mathrm{~nm}^{3}(a=18 \mathrm{~nm})$, the concentration of 2-(trifluoromethyl)acrylic acid in the crystal lattice was calculated as 155.6 $\mathrm{mM}$, which is very similar to its concentration $(179.9 \mathrm{mM})$ in the soaking solution.

\section{Monitoring crystal expansion-contraction using light microscopy.}

Single crystals were transferred with a mounted CryoLoop onto a glass slide with a microscopic ruler (OMAX). All images and videos were obtained on an SZX7 (Olympus) microscope equipped with an Infinity 1 charge-coupled device (CCD; Lumenera). For crystals that had not been polymerized, $10 \mu \mathrm{l}$ of the polymerization solution was carefully added to minimize crystal movement. This solution was removed before water addition. For previously polymerized crystals, water (Milli-Q $30 \mu \mathrm{l}$ ) was added and crystal expansion was observed over 5-20 min. To initiate crystal contraction, water was replaced with a solution containing either $4 \mathrm{M} \mathrm{NaCl}$ or $1 \mathrm{M} \mathrm{CaCl}_{2}$. This expansion-contraction cycle could be repeated at least eight times for a crystal if $\mathrm{NaCl}$ was used to induce crystal contraction. Crystal size was determined by measuring the edge length of a facet relative to the microscopic ruler using the Fiji image processing package. 


\section{Monitoring crystal expansion using confocal microscopy.}

Crystals containing polymer precursors were prepared as described above. One of these crystals was transferred onto a glass slide and imaged on a confocal microscope. After capturing an initial image, the crystal was polymerized in $10 \mu$ of the polymerization solution, and its expansion in $30 \mu \mathrm{l}$ water was monitored. DIC images were captured at different time intervals with a 100-ms exposure until the crystal was no longer visible.

\section{Quantification of protein release during expansion.}

Large-scale crystallization of ferritin was carried out as described above. Once crystals fully matured, the well solution was replaced with $100 \mu \mathrm{l}$ of the polymer precursor solution. After $12 \mathrm{~h}$, the crystals were all combined into a single Eppendorf tube and $500 \mu \mathrm{l}$ of the polymerization solution was added. Crystals were expanded by replacing the polymerization solution with $1 \mathrm{ml}$ water. During this experiment, aliquots $(100 \mu \mathrm{l})$ of the protein solution were removed and replaced with $100 \mu \mathrm{l}$ of water, and each aliquot was used to determine the protein concentration using the Bradford assay.

\section{Multi-crystal expansion monitored using SAXS.}

Crystals for multi-crystal small-angle X-ray scattering were prepared as described above and transferred into the polymer precursor solution. A large number $(n>100)$ of crystals were transferred to an Eppendorf tube. After the crystals had settled at the bottom, they were transferred, along with $50 \mu \mathrm{l}$ of solution, into a $1.5-\mathrm{mm}$ quartz capillary (Hampton). Crystals in capillaries were analysed at beamline 5-ID-D of the Advanced Photon Source (Argonne National Laboratory). Data were collected using collimated X-ray radiation ( $0.7293 \AA$, 17 $\mathrm{keV}$ ) calibrated with both a glassy carbon standard and a silicon diffraction grating. After the sample was mounted on the instrument, a thin tube (with a diameter of $0.51 \mathrm{~mm}$ ) was inserted into the capillary to facilitate the addition of $50 \mu$ of solution with a syringe injector during X-ray exposure. The injected solution contained a more concentrated polymerization solution without $\mathrm{NaCl}$ (2\% APS and 2\% TEMED) in water. After the first exposure, the solution was injected, and an image with a 1-s X-ray exposure was collected every $30 \mathrm{~s}$. Peaks corresponding to the original lattice were visible throughout the process, indicating that some of the crystals in the bulk sample did not expand. This is probably due to limited solvent diffusion or incomplete polymerization within the capillary tubes used for the SAXS experiments. It is important to note that in this procedure, 'polymerized' crystals immediately began expanding upon the commencement of data collection. The reason for this experimental strategy (instead of polymerization in a high-ionic-strength solution, followed by the initiation of expansion through lowering the ionic strength) is that it was not possible to sufficiently dilute the high-ionic-strength polymerization solution in the thin capillary tubes used for SAXS (which cannot accommodate addition of large volumes of solution) to enable expansion.

Scattered radiation was detected using a CCD area detector and onedimensional scattering data were obtained through the azimuthal averaging of the two-dimensional data to produce plots of the scattering intensity as a function of the scattering vector length, $q=4 \pi \sin (\theta / \lambda)$, where $\theta$ is one-half of the scattering angle and $\lambda$ is the wavelength of the $\mathrm{X}$-rays used. 
Analysis of the one-dimensional data was performed using the powder diffraction processing software JADE (MDI) or Origin (OriginLab).

\section{Multi-crystal SAXS at elevated temperatures.}

Large-scale crystallization of ferritin was performed as described above. The crystallization solution was removed, and ferritin crystals were resuspended in either the polymer precursor solution or a buffered solution containing $25 \mathrm{mM}$ HEPES (pH 7.0) and $30 \mathrm{mM} \mathrm{CaCl}_{2}$. After $72 \mathrm{~h}$, the polymer precursor soaking solution was replaced with the polymerization solution. After $10 \mathrm{~min}$, this was also replaced with a buffered solution containing $25 \mathrm{mM}$ HEPES ( $\mathrm{pH}$ 7.0), $4 \mathrm{M} \mathrm{NaCl}$ and $30 \mathrm{mM} \mathrm{CaCl}_{2}$. Both samples, containing either native ferritin crystals or the crystal-hydrogel hybrids, were transferred into 1.5-mm quartz capillaries (Hampton). Data were collected at beamline 4-2 of SSRL using collimated X-ray radiation (1.1271 Å, $11 \mathrm{keV}$ ) calibrated with a silver behenate standard. The samples were heated using a custom-built thermal stage operating at $1{ }^{\circ} \mathrm{C} \mathrm{min}^{-1}$, and images with a 1-s X-ray exposure were collected every minute. Scattered radiation was detected using a Pilatus3 X 1M detector (Detectris) and processed as described above.

\section{Single-crystal SAXS.}

Crystals containing the polymer precursors were prepared and polymerized using the polymerization solution as described above, and were analysed at SSRL (beamline 4-2). Single crystals were harvested with a mounted CryoLoop and transferred into a $2 \mathrm{M} \mathrm{NaCl}$ solution in the 400- $\mu \mathrm{m}$-diameter central well of a custom-made microfluidic chip (Extended Data Fig. 8a, b). The microfluidic chip was sealed with a coverslip, attached to a syringe injector and mounted on beamline 4-2 at SSRL for data collection. Data were collected using collimated X-ray radiation $(1.127 \AA, 11 \mathrm{keV})$ calibrated with a silver behenate standard. Water was injected into the microfluidic chip at $1 \mu \mathrm{s} \mathrm{s}^{-1}$ to initiate expansion, and 0.5 -s-exposure images were taken every about $2.5 \mathrm{~s}$ for $4 \mathrm{~min}$. After the data acquisition for crystal expansion was complete, the process was repeated-in the order $4 \mathrm{M} \mathrm{NaCl}$, water, 1 $\mathrm{M} \mathrm{CaCl}_{2}$, water-to monitor repeated contraction and expansion processes. Data were collected using a Pilatus 3 X 1M detector (Detectris). The unit-cell parameters were determined by calculating the radial distance of individual reflections, after fitting the spot intensity to a two-dimensional Gaussian surface.

\section{Single-crystal XRD at room temperature.}

Crystals containing polymer precursors were prepared and imaged using light microscopy as described above. A single crystal was transferred onto a MicroMount precision tool (MiTeGen) with a 100- $\mu$ m aperture and sealed with a MicroRT capillary (MiTeGen). Data were acquired on an APEX II CCD diffractometer (Bruker) using $\mathrm{Cu} \mathrm{K} a$ radiation (1.5418 $\AA$ ) at $295 \mathrm{~K}$. Three images (60-s exposure) were collected at rotation angles $\varphi=0^{\circ}, 60^{\circ}$ and $120^{\circ}$. The crystal was removed from the instrument and soaked in $10 \mu \mathrm{l}$ of the polymerization solution for $2 \mathrm{~min}$. The crystal was transferred onto a microscopic ruler and $30 \mu \mathrm{l}$ of water was added. Crystal expansion was measured over $3 \mathrm{~min}$. This crystal was returned to the MicroMount with the MicroRT capillary and an identical three-image dataset was collected. This process was repeated using $30 \mu \mathrm{l}$ of a $1 \mathrm{M} \mathrm{CaCl} 2$ solution. After the 
crystal had contracted (1 min), another three-image dataset was collected. Images were analysed with the Apex III software (Bruker).

\section{Single-crystal XRD at $100 \mathrm{~K}$.}

Crystal-hydrogel hybrids were prepared and imaged using light microscopy as described above. Two crystals were harvested, $30 \mu \mathrm{l}$ of water was added, and crystal expansion was monitored over $5 \mathrm{~min}$ for both crystals. After $5 \mathrm{~min}$, the water was removed and $30 \mu \mathrm{l}$ of either a solution containing $1 \mathrm{M} \mathrm{CaCl}_{2}$ (crystal A) or $4 \mathrm{M} \mathrm{NaCl}$ (crystal B) was added. Crystal B was re-expanded in $30 \mu \mathrm{l}$ water. After $5 \mathrm{~min}$, the water was replaced with $30 \mu \mathrm{l}$ of a $1 \mathrm{M} \mathrm{CaCl}_{2}$ solution to contract crystal $\mathrm{B}$. After contraction, both crystals were cryoprotected in perfluoropolyether (Hampton) and frozen in liquid $\mathrm{N}_{2}$. Single-crystal XRD data for the contracted ferritin crystals were collected at $100 \mathrm{~K}$ at beamline 9-2 of SSRL using 0.98 - $\AA$ radiation. The data were integrated using iMosflm ${ }^{34}$ and scaled with Aimless ${ }^{35}$ (Extended Data Table 1). The structures for crystal A and crystal B were determined at resolutions of $1.06 \AA$ and $1.13 \AA$, respectively. Molecular replacement was performed with Phaser ${ }^{36}$ using the HuHF structure (PDB ID, 5CMQ) as a search model. Rigid-body, positional, anisotropic thermal and atom-occupancy refinements were carried out using Phenix ${ }^{37}$. Coot $^{38}$ was used for iterative manual model building. The interstitial solvent content was calculated by subtracting the solvent volume of each crystal from the volume of the inner cavity of ferritin (calculated using VOIDOO) ${ }^{39}$. All figures were produced with Pymol $^{40}$.

\section{Nanoindentation measurements of crystals.}

The mechanical properties of the native ferritin crystals and the crystal-hydrogel hybrids were determined using a Hysitron TI 950 Triboindenter test instrument (Bruker). All crystals were dried before the indentation experiments. A Berkovich probe (TI-0039, 142.3, $100 \mathrm{~nm}$ tip radius) was used to determine the hardness and reduced modulus of the native crystals and crystal-hydrogel hybrids. Experiments were conducted in displacement control mode using a displacement of $1,000 \mathrm{~nm}$.

\section{Preparation of iron-loaded ferritin.}

Iron-loaded ferritin was prepared by adding $10.8 \mathrm{ml}$ of $10 \mathrm{mM}\left(\mathrm{NH}_{4}\right)_{2} \mathrm{Fe}\left(\mathrm{SO}_{4}\right)_{2}$ over $2 \mathrm{~h}$ to $144.8 \mathrm{ml}$ of a vigorously stirring solution containing $1 \mu \mathrm{M}$ ferritin, $15 \mathrm{mM}$ Tris (pH 7.4) and $150 \mathrm{mM} \mathrm{NaCl}$. Subsequently, the solution was stirred for an additional hour before being concentrated to about $3 \mathrm{ml}$ using a 10-kDa Amicon membrane. A 10DG column (Bio-Rad) was used to remove any unbound iron. The iron content was assessed using a 2-2 'bipyridine-based colorimetric assay ${ }^{41}$ and the protein concentration was determined using the Bradford assay. Each ferritin cage contained about $800 \mathrm{Fe}$ atoms.

\section{Formation of core-shell ferritin crystals.}

Expandable core/expandable shell crystals. Mature ferritin crystals were transferred to a buffered solution containing $25 \mathrm{mM}$ HEPES (pH 7.0), $30 \mathrm{mM} \mathrm{CaCl}_{2}$ and $1.9 \mathrm{mM} \mathrm{(1} \mathrm{mg} \mathrm{ml}$ ${ }^{-1}$ ) 5-(and 6)-carboxytetramethyl rhodamine succinimidyl ester (NHS-rhodamine; Thermo Fisher Scientific). After soaking for $12 \mathrm{~h}$, an individual crystal was removed and washed 
three times in a buffered solution containing $25 \mathrm{mM}$ HEPES (pH 7.0) and $30 \mathrm{mM} \mathrm{CaCl}_{2}$ to remove unbound NHS-rhodamine. The crystal was transferred to a well containing $10 \mu \mathrm{l}$ of $12.5 \mu \mathrm{M}$ ferritin, $25 \mathrm{mM}$ HEPES (pH 7.0) and $6 \mathrm{mM} \mathrm{CaCl}_{2}$. A transparent layer of ferritin formed around the rhodamine-labelled ferritin crystal over $12 \mathrm{~h}$ (creating a red core and a transparent shell). This crystal was soaked in a polymer precursor solution and polymerized as described above to yield an expandable core/expandable shell crystal.

Fixed core/expandable shell crystals. Fixed core/expandable shell ferritin crystals were prepared similarly to the expandable core/expandable shell crystals described above. The only difference was that after the rhodamine labelling step, the crystal was transferred into a solution containing $2.5 \%$ (v/v) glutaraldehyde, $25 \mathrm{mM}$ HEPES (pH 7.0) and $30 \mathrm{mM} \mathrm{CaCl}_{2}$. After $12 \mathrm{~h}$, the crystal was washed five times with water to remove unbound glutaraldehyde, followed by the epitaxial growth of the transparent layer of ferritin crystals on top of the core layer in a fresh crystallization solution containing $12.5 \mu \mathrm{M}$ ferritin. This crystal was then soaked in a polymer precursor solution and polymerized as described above to yield a fixed core/expandable shell crystal.

\section{Data availability.}

Crystal structures have been deposited in the Research Collaboratory for Structural Bioinformatics Protein Data Bank under accession codes 6B8F (ferritin-polymer hybrid crystal 1; https://www.rcsb.org/struc-ture/6b8f) and 6B8G (ferritin-polymer hybrid crystal 2; https://www.rcsb.org/structure/6b8g). Raw data are available from F.A.T. 


\section{Extended Data}

\begin{tabular}{cccc}
\hline $\mathrm{pH}$ & 6.0 & 7.0 & 7.5 \\
\hline zeta potential & $-5.54 \pm 0.14 \mathrm{mV}$ & $-6.53 \pm 0.37 \mathrm{mV}$ & $-7.31 \pm 0.12 \mathrm{mV}$ \\
\hline
\end{tabular}

b

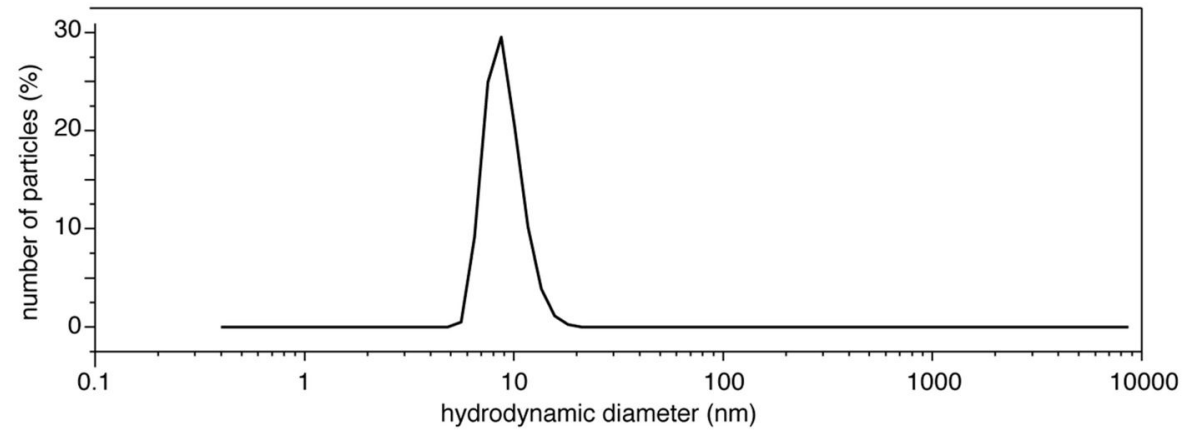

c
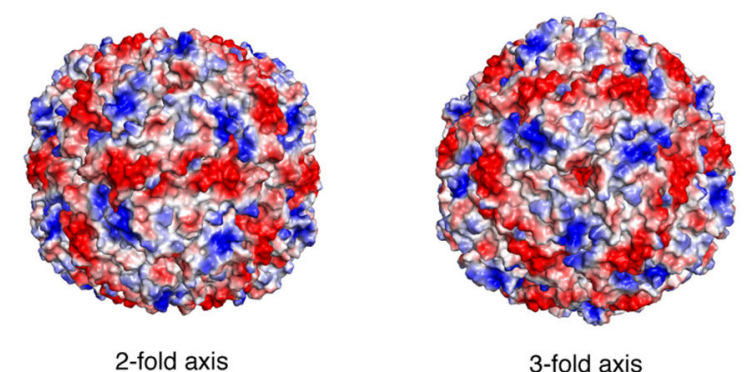

3-fold axis

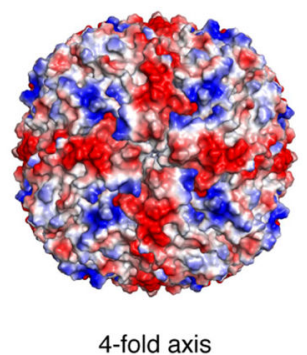

Extended Data Fig. 1 |. Distribution of electrostatic charge on the surface of ferritin and size distribution of ferritin in solution.

a, $\mathrm{pH}$-dependent zeta potentials of ferritin, determined by dynamic-light-scattering measurements. b, Dynamic-light-scattering profile of ferritin $(200 \mu \mathrm{M})$ in a solution of 50 $\mathrm{mM}$ HEPES ( $\mathrm{pH}$ 7.0). c, Representation of the electrostatic charge distribution on the ferritin surface, as viewed along the two-, three- and four-fold symmetry axes. Positive $\left(+5 k_{B} T / e\right)$ and negative $\left(-5 k_{B} T / e\right)$ charges are shown in blue and red, respectively. $k_{\mathrm{B}}$, Boltzmann constant; $e$, electron charge. 

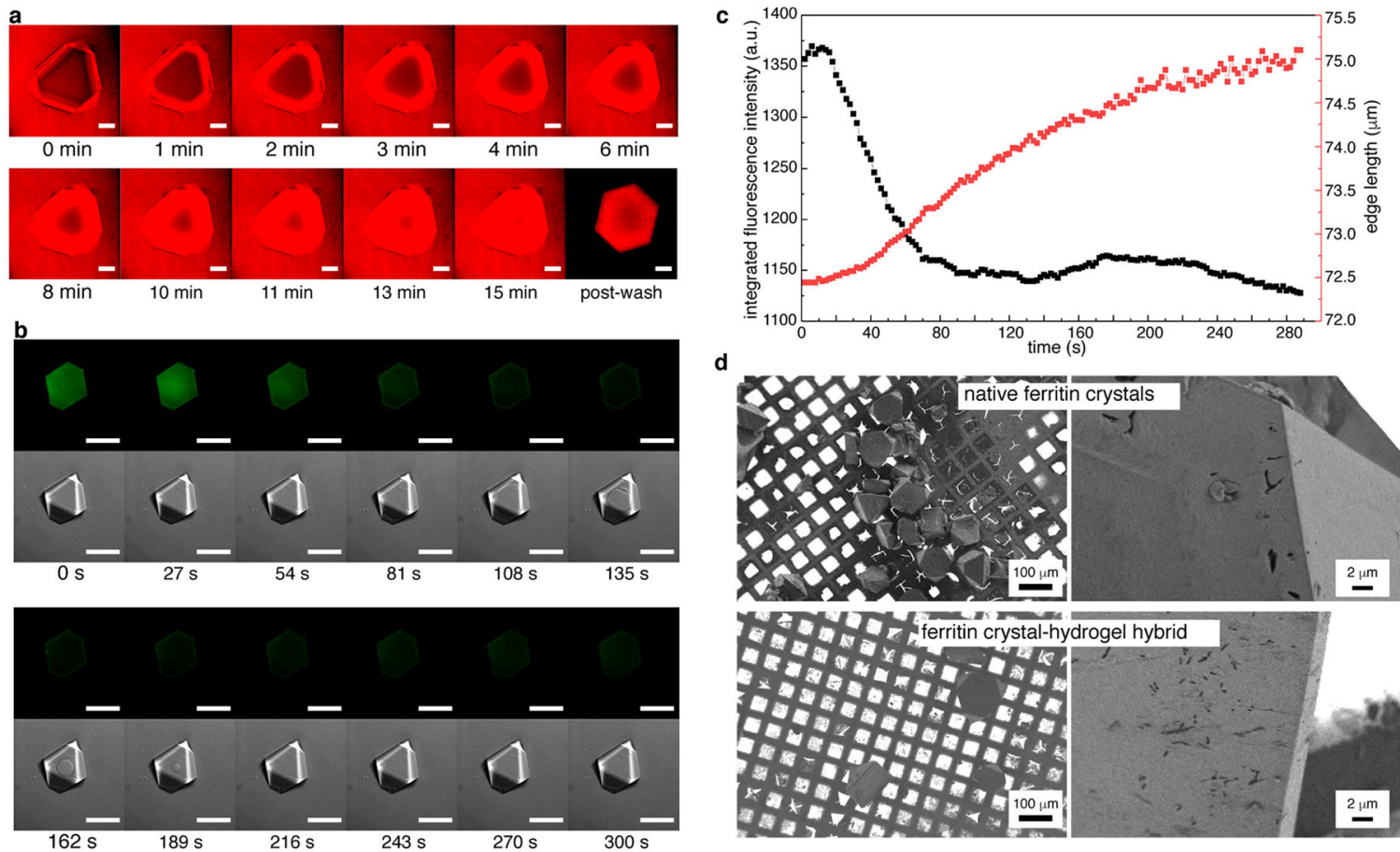

Extended Data Fig. 2 |. Molecular diffusion and polymerization in ferritin crystals, monitored using confocal microscopy.

a, Diffusion of rhodamine B into a ferritin crystal over $15 \mathrm{~min}$. b, c, In crystallo polymerization of the hydrogel network, monitored through the decrease of integrated pyranine fluorescence (green fluorescence channel). The corresponding bright-field (DIC) images show the diffusion of the aqueous $\mathrm{NaCl}$ solution into the crystal. The ring-shaped diffusion front becomes evident at time $t=108 \mathrm{~s}$ and disappears by $t=216 \mathrm{~s}$. The crystal expands by approximately $5 \%$ (edge length) during polymerization. Scale bars in a and b correspond to $100 \mu \mathrm{m}$. d, Scanning electron microscopy images of native ferritin crystals (top) and crystal-hydrogel hybrids (bottom). 
a

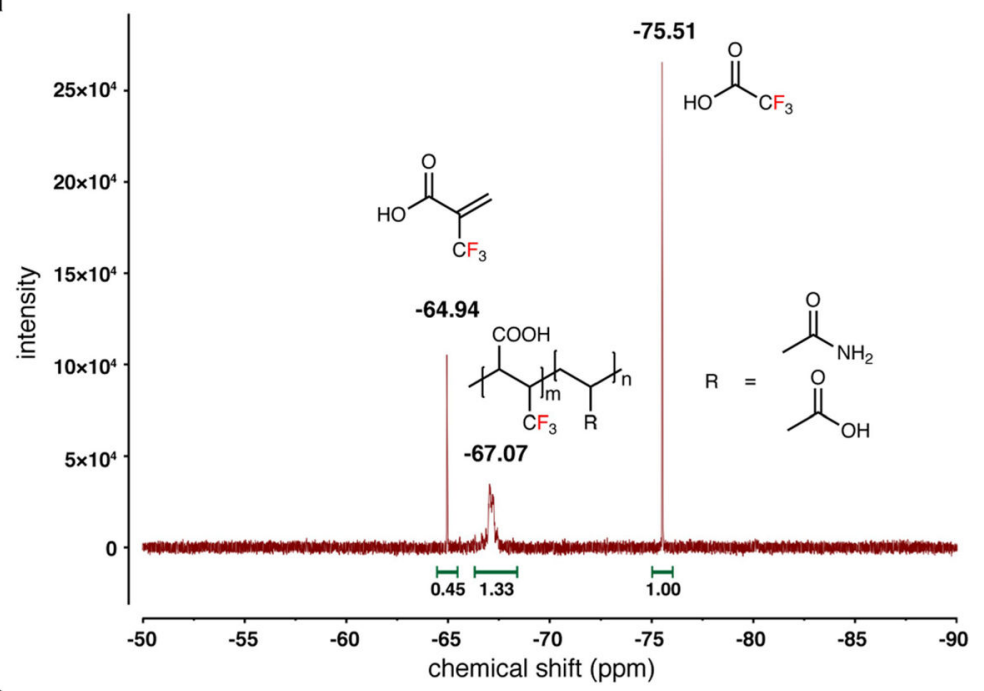

b

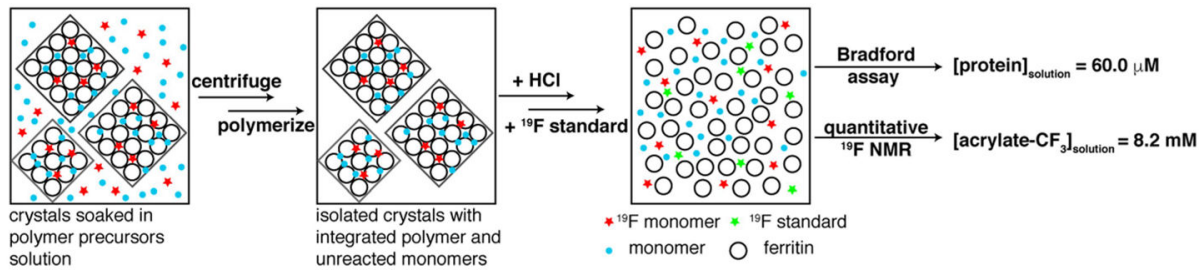

Extended Data Fig. 3 |. Quantification of an acrylic acid analogue using 19FNMR.

a, ${ }^{19}$ F-NMR spectrum, showing peak assignments for the trifluoroacetic acid standard, free 2-(trifluoromethyl)acrylic acid, and 2-(trifluoromethyl)acrylic acid incorporated into the polymer. b, Diagram illustrating the experimental protocol for the quantification of 2(trifluoromethyl)acrylic acid uptake into the crystal lattice. The concentration of the 2(trifluoromethyl)acrylic acid in the crystal lattice $(155.6 \mathrm{mM})$ is approximately the same as its concentration in the soaking solution (see Methods for details). 


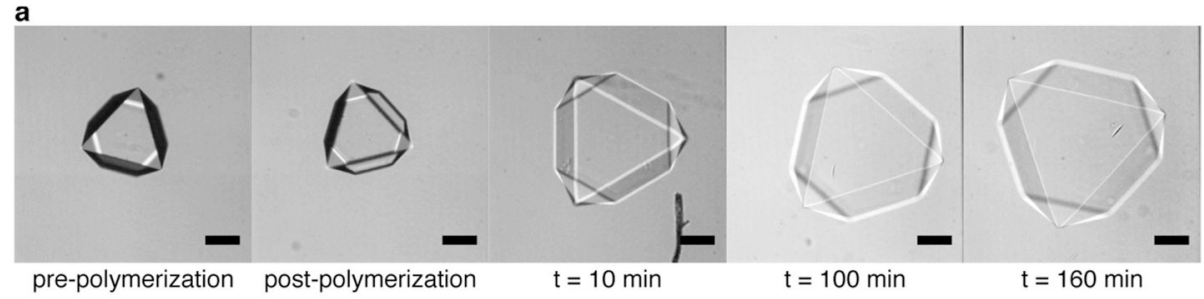

b
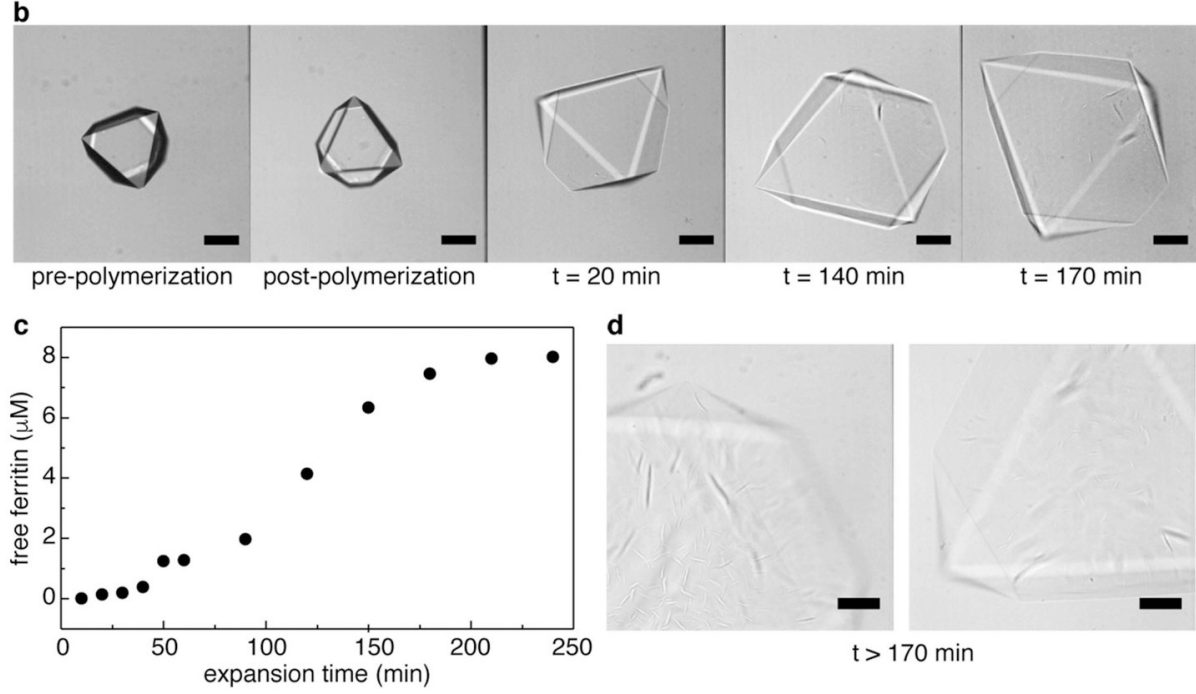

Extended Data Fig. 4 |. Isotropic hyperexpansion of ferritin crystal-hydrogel hybrids.

a, b, Continuous expansion of two different crystal-hydrogel hybrids in deionized water, monitored using confocal microscopy. Crystal facets are still discernible after expansion for more than $2 \mathrm{~h}$. Scale bars correspond to $100 \mu \mathrm{m}$. c, Ferritin release into the solution from expanding crystal-hydrogel hybrids $(n>10,000)$ over about 4 h. Negligible ferritin release is observed until about $1 \mathrm{~h}$. Protein concentrations were determined using the Bradford assay. d, Confocal microscopy images of highly expanded crystal-hydrogel hybrids, showing the structural deterioration of the facets and the edges. 

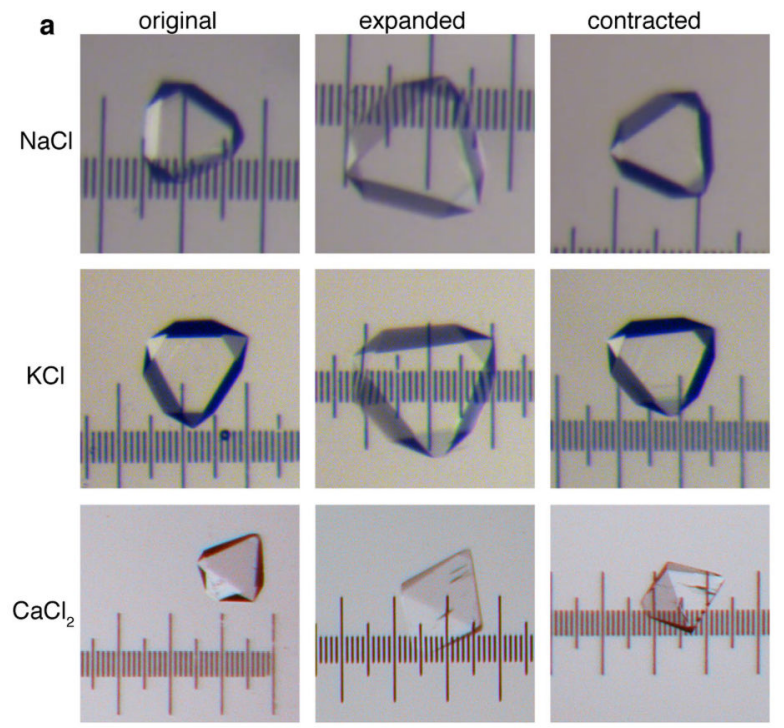

b $\mathrm{LiCl}$
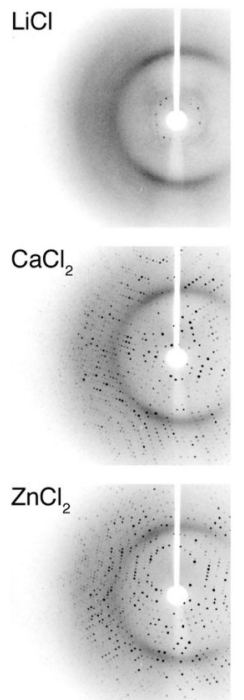

$\mathrm{NaCl}$

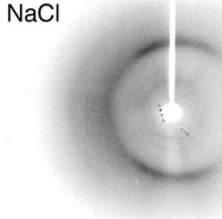

$\mathrm{MgCl}_{2}$

$\mathrm{NiCl}_{2}$

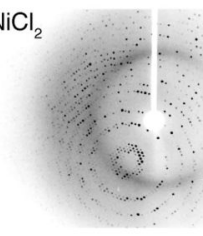

$\mathrm{KCl}$
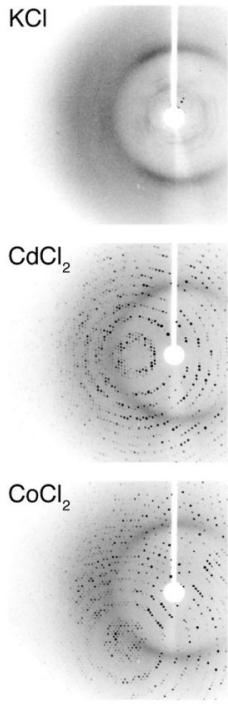

Extended Data Fig. 5 |. Expansion and contraction behaviour of crystal-hydrogel hybrids in the presence of different metal ions.

a, Light micrographs of the crystal-hydrogel hybrids at different stages of expansion and contraction in response to different metal ions. b, XRD patterns ( $T=273 \mathrm{~K}$ ) of expanded crystal-hydrogel hybrids, acquired upon contraction with different metal ions. Contraction with divalent cations $(\mathrm{Ca}, \mathrm{Mg}, \mathrm{Cd}, \mathrm{Zn}, \mathrm{Ni}$ and $\mathrm{Co}$ ) reproducibly leads to the recovery of the full atomic-level order, whereas contraction with monovalent cations ( $\mathrm{Li}, \mathrm{Na}$ and $\mathrm{K}$ ) only reinstates low-order diffraction peaks. 
13

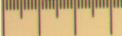

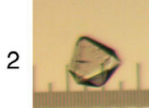

3

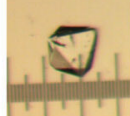

4

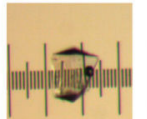

5

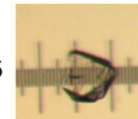

6
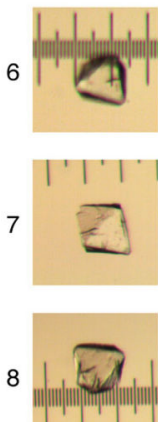

preexpansion
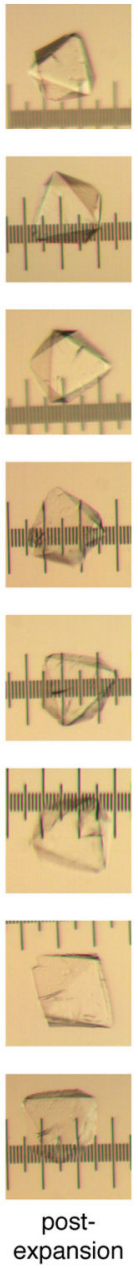
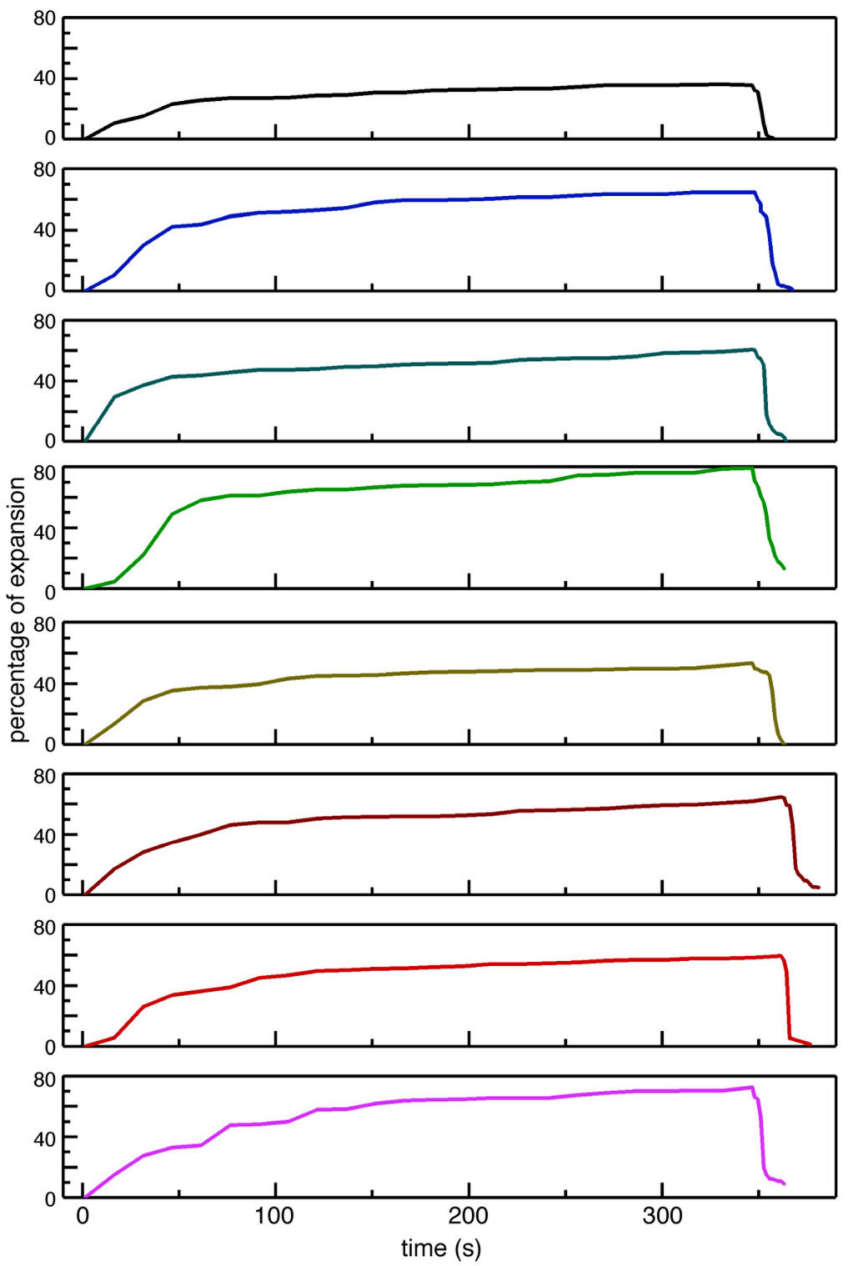

Extended Data Fig. 6 |. Successive expansion-contraction cycles for a single ferritin crystalhydrogel hybrid.

Light micrographs of a hybrid crystal at pre- and post-expansion stages in each cycle are shown on the left, and the corresponding changes in edge length upon expansion-contraction are shown on the right. The separation between the major ticks of the ruler is $100 \mu \mathrm{m}$. The crystal expands to a lesser extent during the first expansion cycle, which we ascribe to residual $\mathrm{CaCl}_{2}$ (which forms strong polymer-polymer and protein-protein interactions) remaining in the solution that is transferred on the loop along with the crystal. The subsequent variability in the rate and extent of expansion is attributed to the different amounts of residual $\mathrm{NaCl}$ transferred in each cycle. 

polymer precursors

post-

polymer precursors

b polymer precursors chemical structure

post-

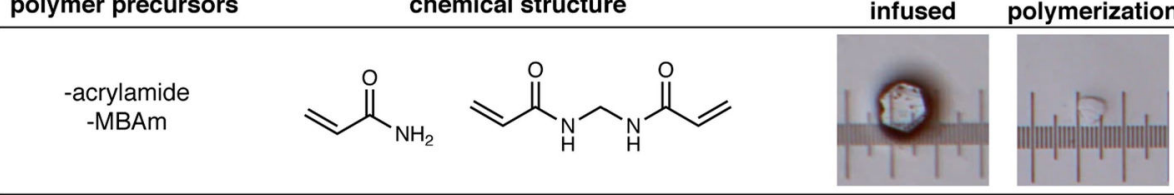

-N-isopropylacrylamide
-MBAm

$\approx \mathrm{H}_{\mathrm{H}}^{\mathrm{O}} \approx \mathrm{Cl}_{\mathrm{H}}^{\mathrm{O}} \mathrm{N}_{\mathrm{H}}^{\mathrm{O}}$
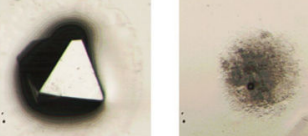

-TRIS-acrylamide -MBAm
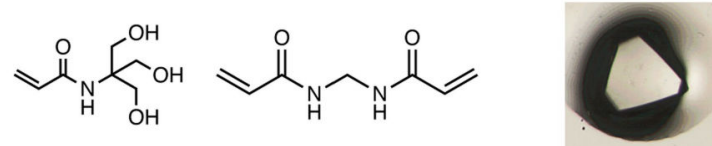

c

polymer
-sodium polyacrylate
$M_{w}=$ ca. $2100 \mathrm{Da}$
$\mathrm{M}_{\mathrm{w}}=\mathrm{ca} .2100 \mathrm{Da}$

chemical structure

polymer soaked hydration

Extended Data Fig. 7 |. Alternative hydrogel formulations.

a, Alternative monomer combinations that yield successful in crystallo polymerization and crystal expansion. b, Monomer combinations that lead to crystal dissolution during polymerization. c, A crystal soaked in a solution containing polyacrylate (molecular weight, $\left.M_{W}=2,100 \mathrm{Da}\right)$ dissolves upon being transferred into water. The separation between the major ticks of the ruler is $100 \mu \mathrm{m}$. MBAm, N,N'-methylenebis(acrylamide). 
a

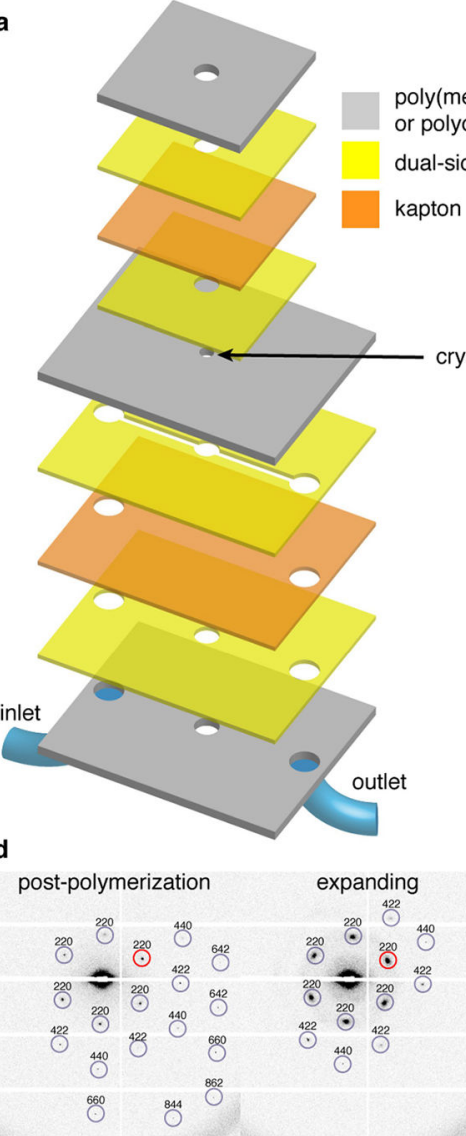

$a=189.95 \AA$

$a=201.32 \AA$ polycarbonate

ual-sided adhesive

ton polyimide film

b

side view

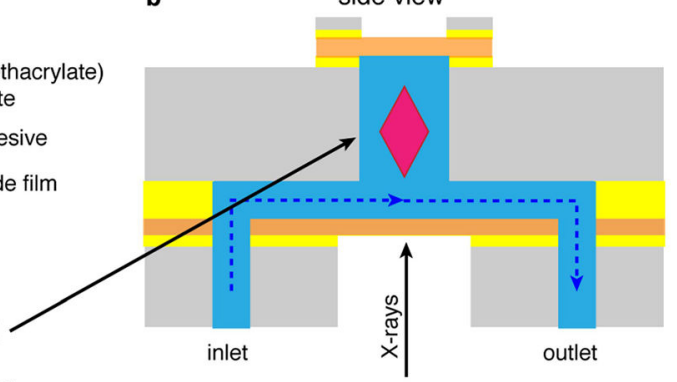

c

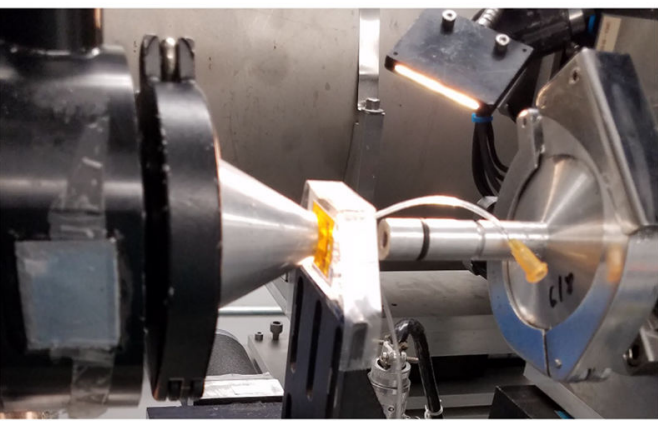

expanding

expanding
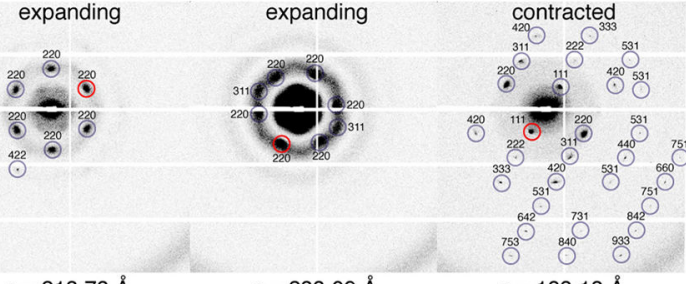

$a=213.73 \AA$

$a=233.09 \AA$

$a=183.13 \AA$

e

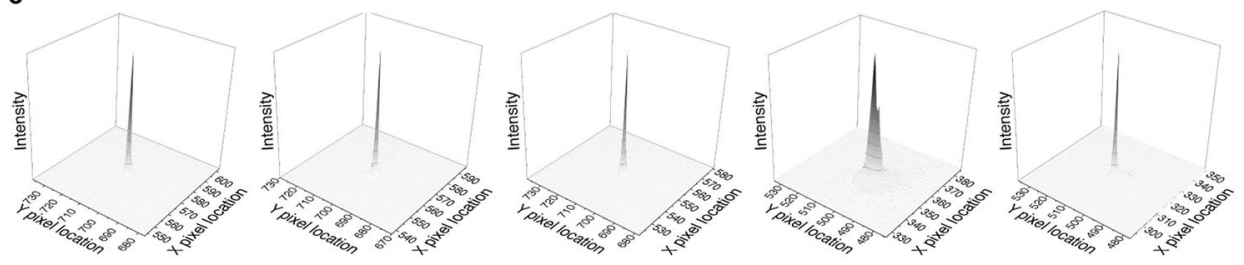

Extended Data Fig. 8 |. SAXS imaging of a single crystal-hydrogel hybrid in a microfluidic chip. a, Schematic diagram of the microfluidic chip. b, Side-view representations of the microfluidic chip. c, Photograph of the microfluidic chip, mounted on beamline 4-2 at SSRL. d, Single-crystal SAXS diffraction patterns observed at different stages of crystal expansion and contraction. The Miller indices of each visible spot are indicated. Reflections with the highest signal-to-noise ratio $(I / \sigma l)$ are circled in red. e, Spot profiles of the highest$I / \sigma I$ reflections indicated in $\mathrm{d}$. 


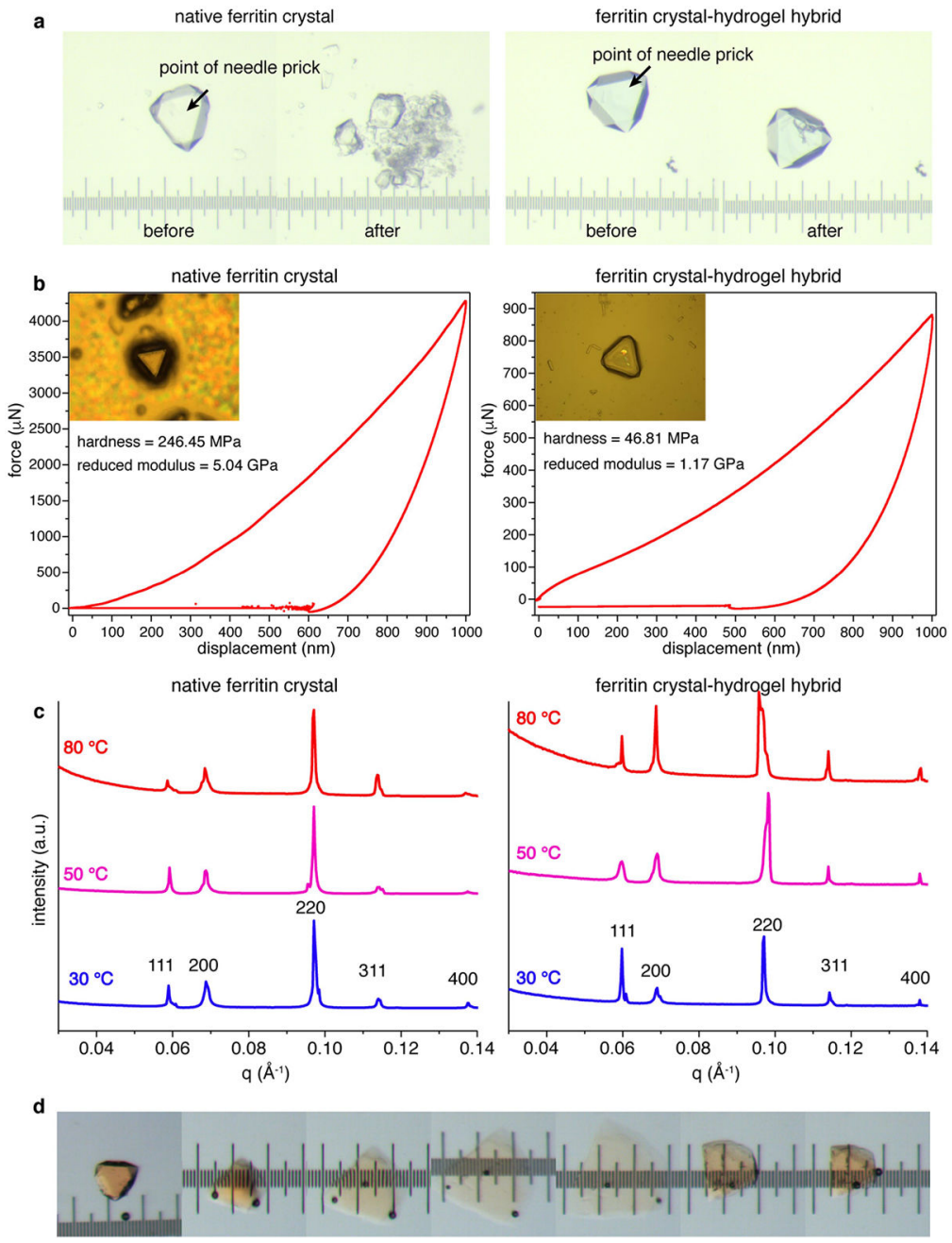

Extended Data Fig. 9 |. Mechanical and thermal properties of ferritin crystal-hydrogel hybrids. a, Light-microscopy images showing the fragmentation of a native ferritin crystal and of a crystal-hydrogel hybrid upon application of external force with a needle at the location indicated with the arrow. The separation between the major ticks of the ruler is $100 \mu \mathrm{m}$. $\mathbf{b}$, Temperature dependence of the SAXS profiles of native ferritin crystals and crystalhydrogel hybrids. The small-angle reflections (that is, periodic order) in both samples are maintained at $80{ }^{\circ} \mathrm{C}$ (the maximal temperature experimentally attainable). c, Determination of the hardness and reduced modulus of native ferritin crystals and crystal-hydrogel hybrids using atomic force microscopy nanoindentation measurements. d, Light-microscopy images showing the expansion and contraction of a crystal-hydrogel hybrid containing Fe-loaded ferritin molecules. The separation between the major ticks of the ruler is $100 \mu \mathrm{m}$.

\section{Extended Data Table 1 |}

$\mathrm{X}$-ray data collection and refinement statistics

$$
\text { Crystal A (6B8F) Crystal B (6B8G) }
$$

Data collection 


\begin{tabular}{|c|c|c|}
\hline & Crystal A (6B8F) & Crystal B (6B8G) \\
\hline Space group & $F 432$ & $F 432$ \\
\hline \multicolumn{3}{|l|}{ Cell dimensions } \\
\hline$a, b, c(\AA)$ & 180.40 & 179.95 \\
\hline$a, \beta, \gamma\left({ }^{\circ}\right)$ & 90 & 90 \\
\hline Resolution $(\AA)$ & $63.65-1.06$ & $63.62-1.13$ \\
\hline Number of unique reflections & 111189 & 92912 \\
\hline Multiplicity & $14.0(2.4)$ & $32.2(13.8)$ \\
\hline$C C 1 / 2$ & $0.999(0.897)$ & $0.999(0.628)$ \\
\hline Rmerge & $0.060(0.222)$ & $0.131(1.131)$ \\
\hline$\langle|/ \sigma|\rangle$ & $24.8(3.2)$ & $19.1(2.3)$ \\
\hline Completeness (\%) & $99.0(86.4)$ & $100(100)$ \\
\hline Average mosaicity $\left({ }^{\circ}\right)$ & 0.17 & 0.31 \\
\hline Total solvent content $(\%)$ & 57.42 & 57.36 \\
\hline Interstitial solvent content $(\%)$ & 39.72 & 39.62 \\
\hline \multicolumn{3}{|l|}{ Refinement } \\
\hline$R_{\text {work }} / R_{\text {free }}$ & $0.0910 / 0.1026$ & $0.1029 / 0.1213$ \\
\hline \multicolumn{3}{|l|}{ Number of atoms } \\
\hline Protein & 1687 & 1699 \\
\hline Ligand/ion & 13 & 13 \\
\hline Water & 340 & 372 \\
\hline \multicolumn{3}{|l|}{$B$-factors $\left(\AA^{2}\right)$} \\
\hline Protein & 8.51 & 9.81 \\
\hline Ligand/ion & 10.19 & 11.96 \\
\hline Water & 21.83 & 23.75 \\
\hline \multicolumn{3}{|l|}{ Root-mean-square deviations } \\
\hline Bond lengths $(\AA)$ & 0.013 & 0.011 \\
\hline Bond angles $\left({ }^{\circ}\right)$ & 1.317 & 1.241 \\
\hline MolProbity ${ }^{44}$ score & 1.20 & 1.38 \\
\hline Clashscore & 4.12 & 6.99 \\
\hline \multicolumn{3}{|l|}{ Ramachandran plot (\%) } \\
\hline Favoured & 98.82 & 98.82 \\
\hline Outliers & 0.00 & 0.00 \\
\hline \multicolumn{3}{|l|}{ Rotamers (\%) } \\
\hline Favoured & 97.35 & 96.88 \\
\hline Poor & 0.53 & 0.52 \\
\hline DPI $(\AA)^{45}$ & 0.011 & 0.015 \\
\hline
\end{tabular}

Numbers in parentheses correspond to the highest resolution shell. Rmerge and CC 1/2 are measurements used to determine an appropriate high-resolution limit for XRD data.

\section{Supplementary Material}

Refer to Web version on PubMed Central for supplementary material.

Nature. Author manuscript; available in PMC 2019 January 16. 


\section{Acknowledgements}

We thank the following colleagues for assistance: R. Alberstein for data processing; A. Rheingold, C. Moore and M. Gembicky for XRD; S. Weigand, T Weiss and I. Rajkovic for SAXS; W.-J. Rappel for confocal microscopy; Z. $\mathrm{Hu}$ for performing the nanoindentation experiments. This work was primarily funded by the US Department of Energy, DOE (BES, Division of Materials Sciences, Biomolecular Materials Program, DE-SC0003844 to F.A.T). Additional funding was provided by NSF (DMR-1602537 to F.A.T for SAXS studies). Crystallographic data were collected at Stanford Synchrotron Radiation Lightsource (SSRL) and the Crystallography Facility of the University of California, San Diego. SAXS data were collected at SSRL and the Advanced Photon Source. SSRL and the Advanced Photon Source are supported by the DOE Office of Science, Office of Basic Energy Sciences under contracts DE-AC02-76SF00515 and DE-AC02-06CH11357, respectively.

\section{References}

1. Hawkins T, Mirigian M, Yasar MS \& Ross JL Mechanics of microtubules. J. Biomech 43, 23-30 (2010). [PubMed: 19815217]

2. Fletcher DA \& Mullins RD Cell mechanics and the cytoskeleton. Nature 463, 485-592 (2010). [PubMed: 20110992]

3. Block SM, Blair DF \& Berg HC Compliance of bacterial flagella measured with optical tweezers. Nature 338, 514-518 (1989). [PubMed: 2648159]

4. Lewis JK, Bothner B, Smith TJ \& Siuzdak G Antiviral agent blocks breathing of the common cold virus. Proc. Natl Acad. Sci. USA 95, 6774-6778 (1998). [PubMed: 9618488]

5. Lok S-M et al. Binding of a neutralizing antibody to dengue virus alters the arrangement of surface glycoproteins. Nat. Struct. Mol. Biol 15, 312-317 (2008). [PubMed: 18264114]

6. Kobatake S, Takami S, Muto H, Ishikawa T \& Irie M Rapid and reversible shape changes of molecular crystals on photoirradiation. Nature 446, 778-781 (2007). [PubMed: 17429396]

7. Kim T, Al-Muhanna MK, Al-Suwaidan SD, Al-Kaysi RO \& Bardeen CJ Photoinduced curling of organic molecular crystal nanowires. Angew. Chem. Int. Edn 52, 6889-6893 (2013).

8. Panda MK et al. Spatially resolved analysis of short-range structure perturbations in a plastically bent molecular crystal. Nat. Chem 7, 65-72 (2015). [PubMed: 25515892]

9. Naumov P, Chizhik S, Panda MK, Nath NK \& Boldyreva E Mechanically responsive molecular crystals. Chem. Rev 115, 12440-12490 (2015). [PubMed: 26535606]

10. Barthelet K, Marrot J, Riou D \& Ferey G A breathing hybrid organic-inorganic solid with very large pores and high magnetic characteristics. Angew. Chem. Int. Edn Engl. 41, 281-284 (2002).

11. Sakata Y et al. Shape-memory nanopores induced in coordination frameworks by crystal downsizing. Science 339, 193-196 (2013). [PubMed: 23307740]

12. Rabone J et al. An adaptable peptide-based porous material. Science 329, 1053-1057 (2010). [PubMed: 20798314]

13. Suzuki Y et al. Self-assembly of coherently dynamic, auxetic, two-dimensional protein crystals. Nature 533, 369-373 (2016). [PubMed: 27135928]

14. Serre $\mathrm{C}$ et al. Role of solvent-host interactions that lead to very large swelling of hybrid frameworks. Science 315, 1828-1831 (2007). [PubMed: 17395825]

15. Worthy A et al. Atomic resolution of structural changes in elastic crystals of copper(ii) acetylacetonate. Nat. Chem 10, 65-69 (2018). [PubMed: 29256512]

16. Mason JA et al. Methane storage in flexible metal-organic frameworks with intrinsic thermal management. Nature 527, 357-361 (2015). [PubMed: 26503057]

17. Couck $\mathrm{S}$ et al. An amine-functionalized MIL-53 metal-organic framework with large separation power for $\mathrm{CO}_{2}$ and $\mathrm{CH}_{4}$. J. Am. Chem. Soc 131, 6326-6327 (2009). [PubMed: 19374416]

18. Chen $\mathrm{Q}$ et al. A controllable gate effect in cobalt(ii) organic frameworks by reversible structure transformations. Angew. Chem. Int. Edn 52, 11550-11553 (2013).

19. Ghosh S \& Reddy CM Elastic and bendable caffeine cocrystals: implications for the design of flexible organic materials. Angew. Chem. Int. Edn 51, 10319-10323 (2012).

20. Commins P, Hara H \& Naumov P Self-healing molecular crystals. Angew. Chem. Int. Edn 55, 13028-13032 (2016). 
21. Tanaka T et al. Phase transitions in ionic gels. Phys. Rev. Lett 45, 1636-1639 (1980).

22. Phadke A et al. Rapid self-healing hydrogels. Proc. Natl Acad. Sci. USA 109, 4383-4388 (2012). [PubMed: 22392977]

23. Holtz JH \& Asher SA Polymerized colloidal crystal hydrogel films as intelligent chemical sensing materials. Nature 389, 829-832 (1997). [PubMed: 9349814]

24. Chen F, Tillberg PW \& Boyden ES Expansion microscopy. Science 347, 543-548 (2015). [PubMed: 25592419]

25. Elliott JE, Macdonald M, Nie J \& Bowman CN Structure and swelling of poly (acrylic acid) hydrogels: effect of $\mathrm{pH}$, ionic strength, and dilution on the crosslinked polymer structure. Polymer 45, 1503-1510 (2004).

26. Theil EC Ferritin: structure, gene regulation, and cellular function in animals, plants, and microorganisms. Annu. Rev. Biochem 56, 289-315 (1987). [PubMed: 3304136]

27. Lawson DM et al. Solving the structure of human $\mathrm{H}$ ferritin by genetically engineering intermolecular crystal contacts. Nature 349, 541-544 (1991). [PubMed: 1992356]

28. Kaya D, Pekcan Ö \& Yilmaz Y Direct test of the critical exponents at the sol-gel transition. Phys. Rev. E 69, 016117 (2004).

29. Strandman S \& Zhu X Self-healing supramolecular hydrogels based on reversible physical interactions. Gels 2, 16 (2016).

30. Denisin AK \& Pruitt BL Tuning the range of polyacrylamide gel stiffness for mechanobiology applications. Appl. Mater. Interfaces 8, 21893-21902 (2016).

31. Huard DJ, Kane KM \& Tezcan FA Re-engineering protein interfaces yields copper-inducible ferritin cage assembly. Nat. Chem. Biol 9, 169-176 (2013). [PubMed: 23340339]

32. Sontz PA, Bailey JB, Ahn S \& Tezcan FA A metal organic framework with spherical protein nodes: rational chemical design of 3D protein crystals. J. Am. Chem. Soc 137, 11598-11601 (2015). [PubMed: 26305584]

33. Bradford MM A rapid and sensitive method for the quantification of microgram quantities of protein utilizing the principle of protein-dye binding. Anal. Biochem 72, 248-254 (1976). [PubMed: 942051]

34. Battye TGG, Kontogiannis L, Johnson O, Powell HR \& Leslie AG iMOSFLM: a new graphical interface for diffraction-image processing with MOSFLM. Acta Crystallogr. D 67, 271-281 (2011). [PubMed: 21460445]

35. Evans PR \& Murshudov GN How good are my data and what is the resolution? Acta Crystallogr. D 69, 1204-1214 (2013). [PubMed: 23793146]

36. McCoy AJ et al. Phaser crystallographic software. J. Appl. Cryst 40, 658-674 (2007). [PubMed: 19461840]

37. Adams PD et al. PHENIX: a comprehensive Python-based system for macromolecular structure solution. Acta Crystallogr. D 66, 213-221 (2010). [PubMed: 20124702]

38. Emsley P, Lohkamp B, Scott WG \& Cowtan K Coot: model-building tools for molecular graphics. Acta Crystallogr. D 66, 486-501 (2010). [PubMed: 20383002]

39. Kleywegt GJ \& Jones TA Detection, delineation, measurement and display of cavities in macromolecular structures. Acta Crystallogr. D 50, 178-185 (1994). [PubMed: 15299456]

40. The PyMOL Molecular Graphics System Version 1.3, https://pymol.org/2/support.html (Schrödinger LLC).

41. Levi S et al. Mechanism of ferritin iron uptake: activity of the H-chain and deletion mapping of the ferro-oxidase site. J. Biol. Chem 263, 18086-18092 (1988). [PubMed: 3192527] 

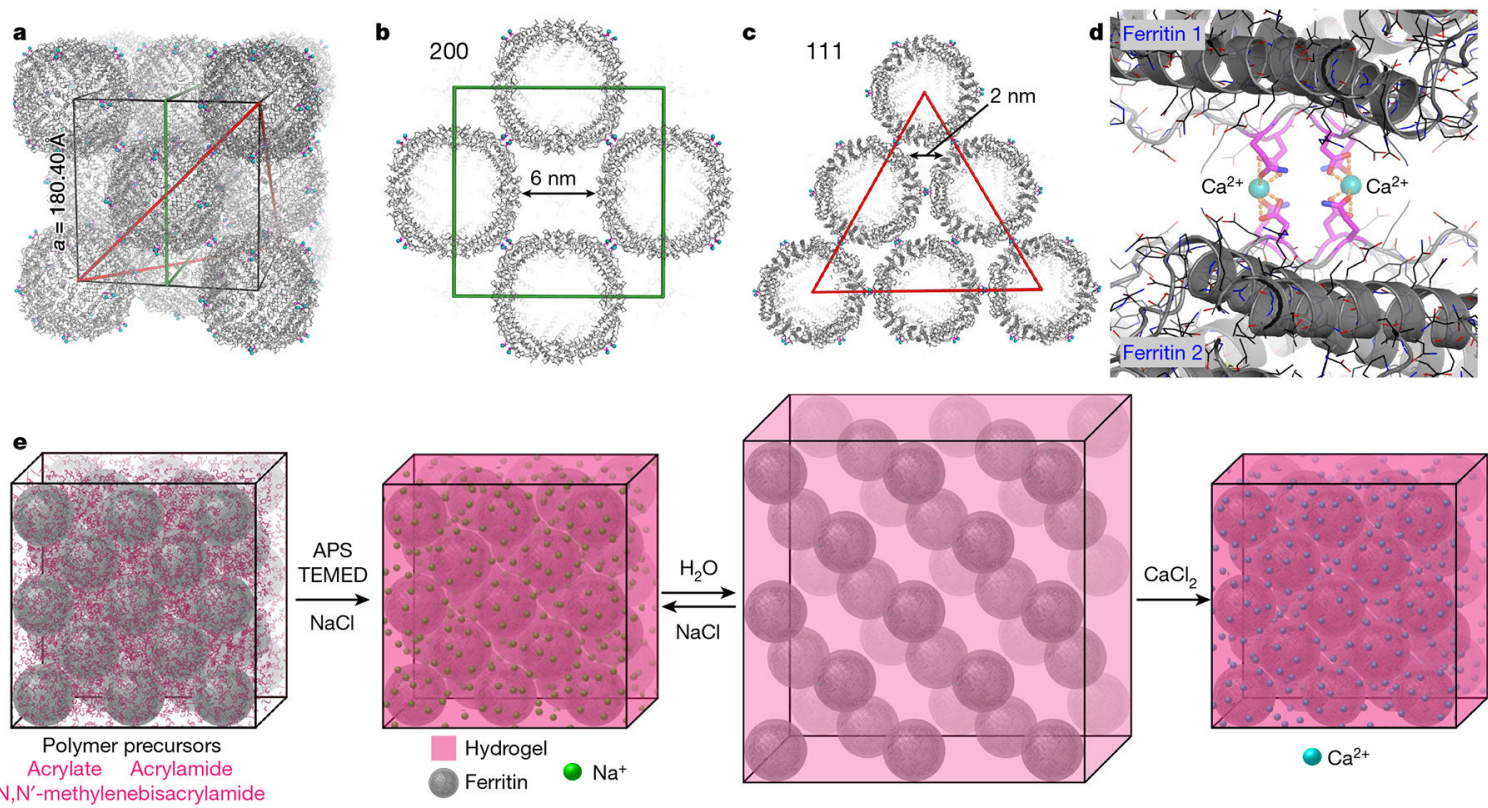

Fig. 1 .

Packing arrangement in ferritin crystals and their expansion-contraction mediated by the infused hydrogel network. a-c, The fcc packing arrangement of ferritin crystals (Protein Data Bank identifier, PDB ID, 6B8F). The unit cell, the 200 plane and the 111 plane are outlined in black, green and red, respectively. $\mathrm{d}, \mathrm{Ca}$-mediated intermolecular interactions between ferritin molecules in the lattice. $\mathrm{Ca}^{2}+$ ions (blue) are coordinated by two pairs of D84 and Q86 side-chains (magenta). e, Schematic representation of the formation, expansion and contraction of ferritin crystal-hydrogel hybrids. 
a
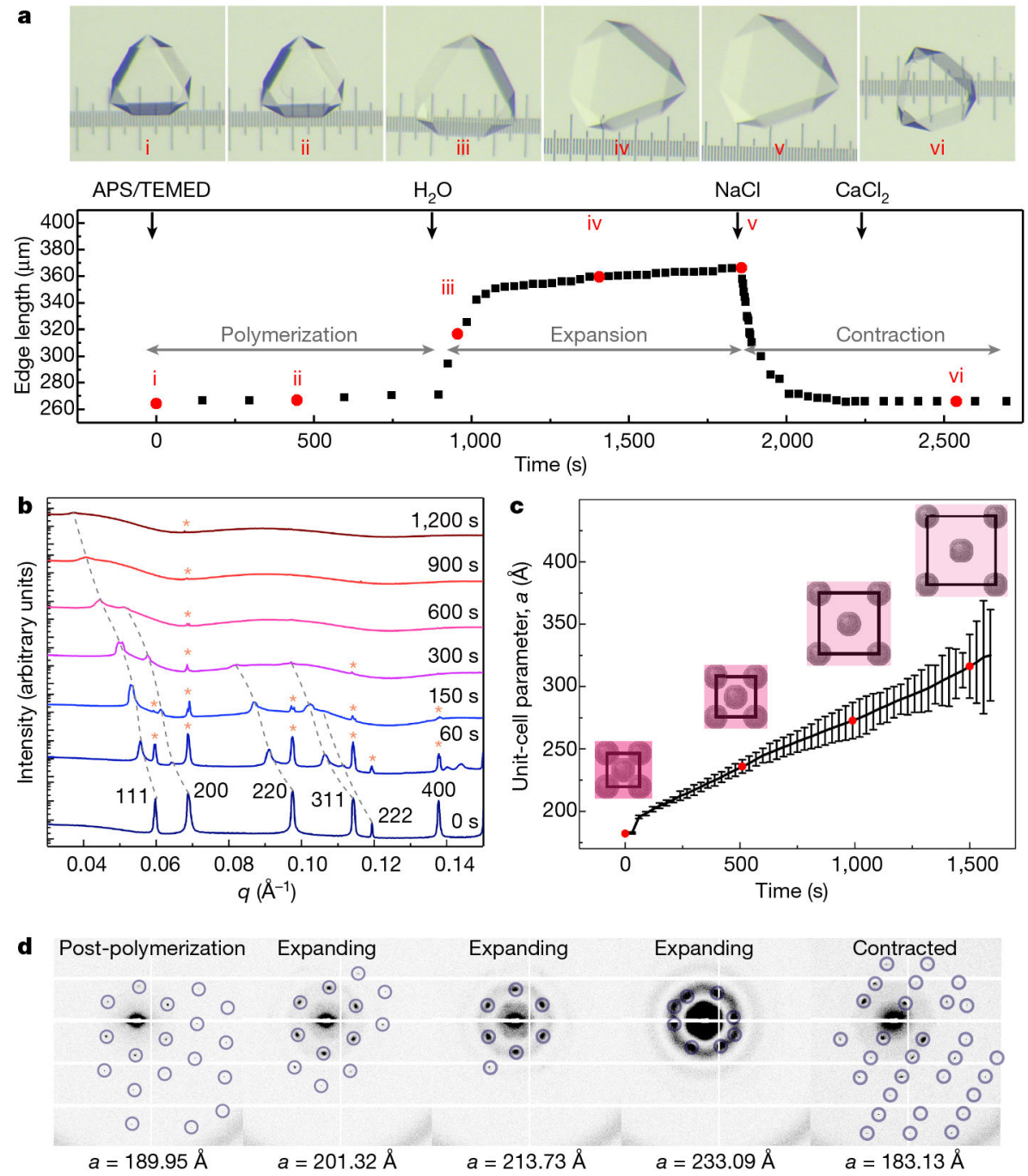

Fig. 2 |.

Characterization of the expansion and contraction behaviour of ferritin crystal-hydrogel hybrids. a, Structural evolution of a ferritin crystal-hydrogel hybrid during the polymerization-expansion-contraction process. Black arrows indicate the addition of different solutions or water to the crystal. The numbered images (i-vi) in the top panels correspond to the selected time points shown as red circles in the bottom panel. The separation between the major ticks of the ruler is $100 \mu \mathrm{m}$. b, SAXS profiles of hydrogelinfused ferritin crystals during lattice expansion, plotted against the scattering vector length $q$. The progression of scattering peaks to lower angles is indicated with grey dashed lines. Peaks corresponding to the original lattice parameters (designated with red asterisks) are visible throughout the process. c, Changes in the unit-cell parameter a during lattice expansion, calculated from the SAXS profiles shown in $b$. The schematics correspond to the red circles and are drawn to scale. The error bars were determined from the full-widths at halfmaximum of the scattering peaks. d, Expansion and contraction of a single crystal, monitored using SAXS. 


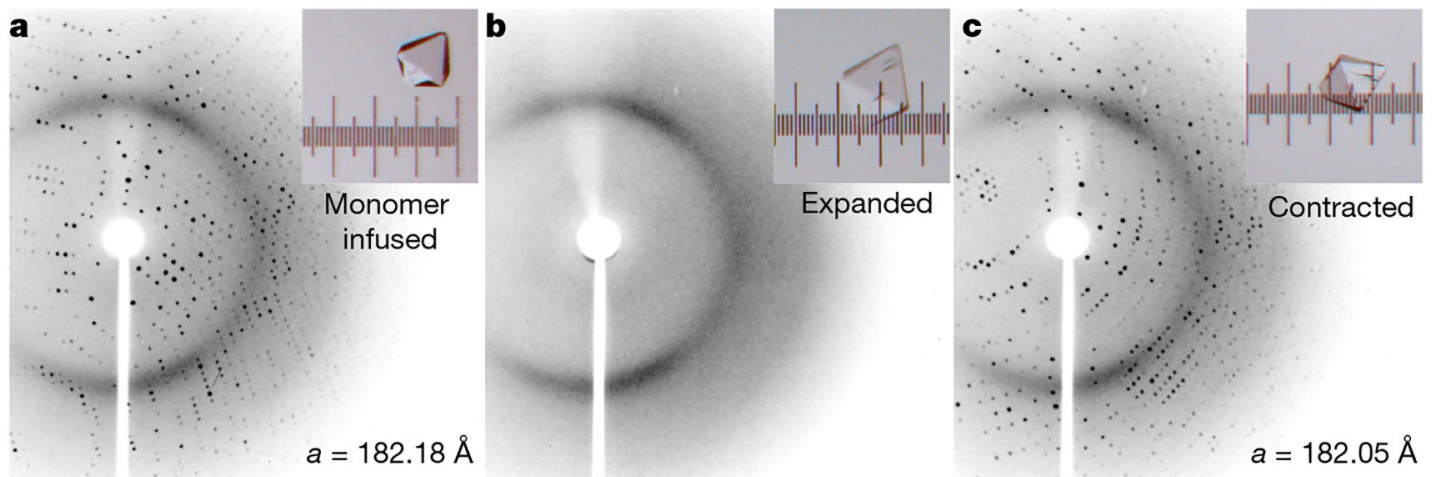

d

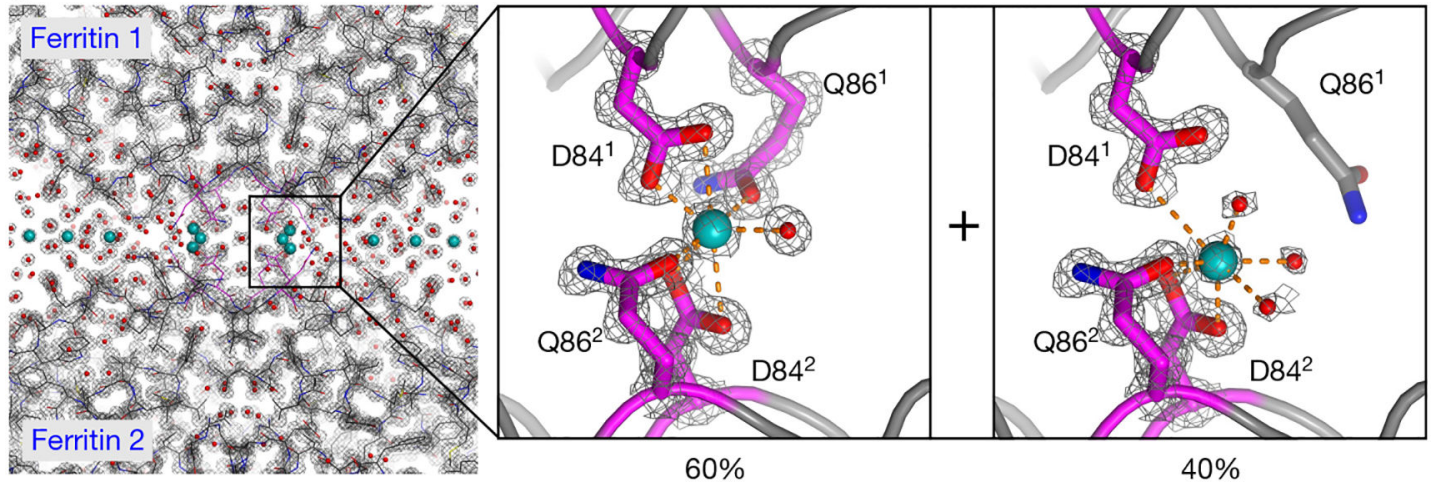

Fig. 3 |.

Atomic-level structural characterization of ferritin crystal-hydrogel hybrids by XRD. a-c, XRD patterns (at temperature $T=293 \mathrm{~K}$ ) of a ferritin crystal infused with polymer precursors (a), after polymerization and expansion (b) and after contraction with $\mathrm{CaCl}_{2}$ (c). Light micrographs of the crystal are shown in the insets; the separation between the major ticks of the ruler is $100 \mu \mathrm{m}$. d, 1.06- $\AA$-resolution structure ( $T=100 \mathrm{~K}$; PDB ID, 6B8F) of the contracted ferritin crystal-hydrogel hybrid, showing the electron density surrounding the Ca-mediated ferritin-ferritin interfaces and highlighting the two observed Ca coordination conformations. The electron density $\left(2 \mathrm{~F}_{\mathrm{o}}-F_{c}\right)$ map (grey) is contoured at $1.5 \sigma$. Water molecules and $\mathrm{Ca}$ ions are shown as red and blue spheres, respectively. 

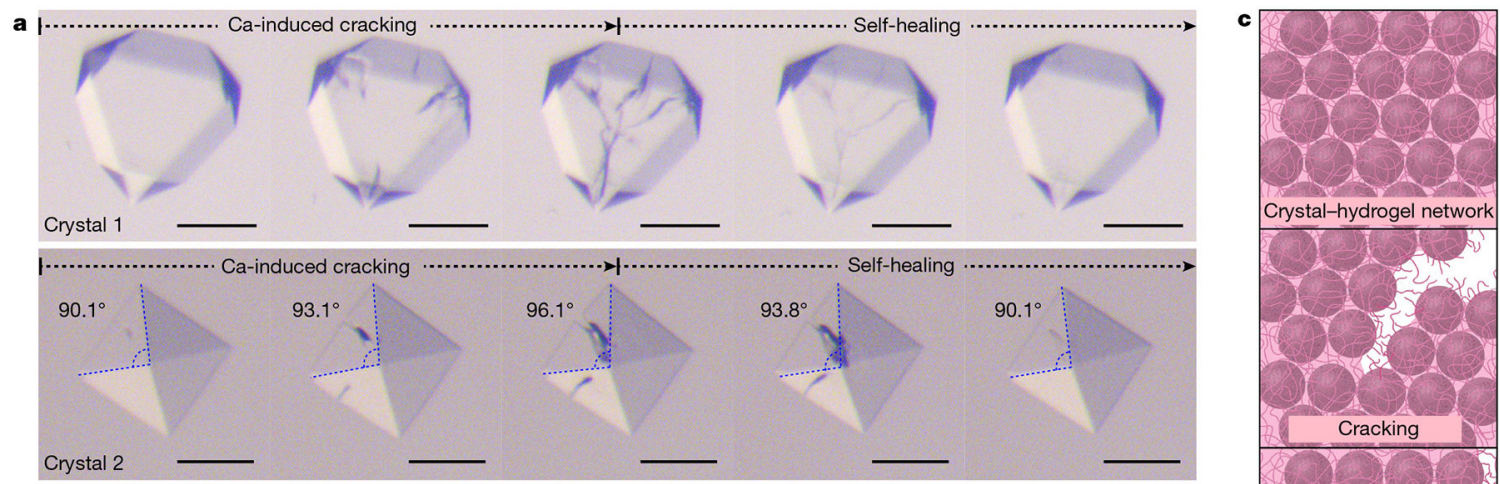

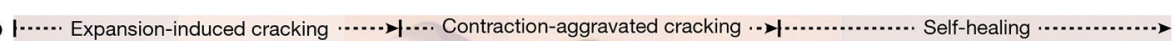
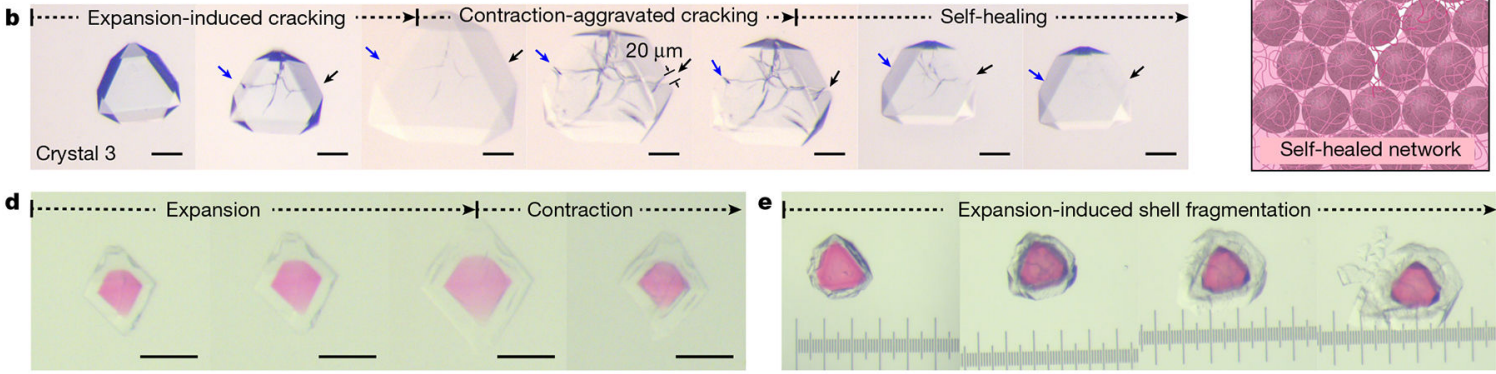

Fig. 4 |.

Self-healing behaviour and functionalization of ferritin crystal-hydrogel hybrids. a, Light microscopy images of crystal-hydrogel hybrids, showing the self-healing of cracks that appear during Ca-induced contraction. b, Extensive cracks can also appear during crystal expansion or during the initial stages of $\mathrm{NaCl}$-induced contraction, but eventually self-heal. The arrows point to the termini of the major crack extending through the crystal. c, Schematic of crack formation and self-healing through the interactions between polymer strands and ferritin molecules. d, Isotropic expansion and contraction of a crystal-hydrogel hybrid with an expandable core and an expandable shell. e, Swelling-induced fragmentation of the shell of a crystal-hydrogel hybrid with a fixed core and expandable shell. All scale bars, as well as the separation between the major ticks of the ruler in e, correspond to a length of $100 \mu \mathrm{m}$. See Supplementary Videos 3 and 4 that accompany this figure. 